NEIP-00-008

hep-th/0003180

\title{
String Theory and Noncommutative Field Theories at One Loop
}

\author{
Adel Bilal, Chong-Sun Chu and Rodolfo Russo \\ Institute of Physics, University of Neuchâtel, CH-2000 Neuchâtel, Switzerland \\ Adel.Bilal@iph.unine.ch \\ Chong-Sun.Chu@iph.unine.ch \\ Rodolfo.Russo@iph.unine.ch
}

\begin{abstract}
By exploiting the boundary state formalism we obtain the string correlator between two internal points on the one loop open string world-sheet in the presence of a constant background $B$-field. From this derivation it is clear that there is an ambiguity when one tries to restrict the Green function to the boundary of the surface. We fix this ambiguity by showing that there is a unique form for the correlator between two points on the boundary which reproduces the one loop field theory results of different noncommutative field theories. In particular, we present the derivation of one loop diagrams for $\phi_{6}^{3}$ and $\phi_{4}^{4}$ scalar interactions and for Yang-Mills theory. From the 2-point function we are able to derive the one loop $\beta$-function for noncommutative gauge theory.
\end{abstract}




\section{Introduction}

Recently, noncommutative field theory has shown up as an effective description of string theory in a certain background [1, 2, 3, 4, 5, 6]. The non-standard commutation relation among space coordinates takes the form

$$
\left[x^{\mu}, x^{\nu}\right]=i \theta^{\mu \nu} \quad \text { with } \mu, \nu \neq 0
$$

where $\theta^{\mu \nu}$ is an antisymmetric real constant matrix of dimension length squared. In the dual language, the algebra of functions is described by the Moyal product

$$
(f * g)(x)=\left.e^{i \frac{\theta^{\mu \nu}}{2} \frac{\partial}{\partial \xi^{\mu}} \frac{\partial}{\partial \zeta^{\nu}}} f(x+\xi) g(x+\zeta)\right|_{\xi=\zeta=0},
$$

which is associative and noncommutative. The noncommutative nature of string theory in the presence of a non-vanishing $F$-field was emphasized in [3, 7]. There it was shown that when quantizing an open string with the boundary condition

$$
\partial_{\sigma} X^{\mu}+\partial_{\tau} X^{\nu} F_{\nu}^{\mu}=0
$$

where $F=B-d A$ is the gauge invariant Born-Infeld field strength, the noncommutativity is an unavoidable feature of open string dynamics. In fact one finds that the coordinates $x^{\mu}$ of the open string endpoints have to satisfy the new relation (1.1) and also the commutation relations for the modes of the string expansion are modified.

Recent interest in noncommutative quantum field theory was boosted by the paper of Seiberg and Witten [6] where it is systematically shown how tree-level open string theory, in the presence of a non-zero $F$-field and of $\mathrm{D} p$-branes, leads to a noncommutative quantum field theory. One of the main observations in [6] was that, in the presence of a F-field, the open string world-sheet Green's function on the boundary of the disk is modified [8]. In particular, Seiberg and Witten showed that in the limit

$$
\begin{gathered}
\alpha^{\prime} \sim \epsilon, \quad F \sim 1 / \epsilon, \\
g_{i j} \sim \epsilon^{2},
\end{gathered}
$$

the tree level amplitudes just consist of a phase factor, which corresponds to the vertices of a noncommutative field theory. The limit $g_{i j} \sim \epsilon^{2}$ is necessary if one wants to kill the string propagators to get an irreducible field theory vertex from an $M$-point string amplitude. In fact, the basic building block in open string theory is the 3-string vertex: thus in order to get higher point interactions (e.g. $\phi^{* n}, n \geq 4$ ), one may sew together a couple of 3 -string vertices and contract some of the propagators in between. This is accomplished by (1.5). Due to some existing confusions in the literature, we feel that it is worthwhile to stress that the "contraction of propagator" (1.5) should be imposed only when necessary and should not always be understood. Indeed, for our purposes of 
obtaining more general field theory amplitudes, it would be wrong to always insist on this limit, as this would kill all the propagators, including the loop propagators. However the other scaling limit (1.4) is necessary to obtain a noncommutative field theory and we will refer to it as the noncommutative limit. In this paper, we will show that by taking the limit (1.4) (and (1.5) only when necessary), one can reproduce from string theory different noncommutative field theories, at the tree and one loop levels.

The noncommutative scalar [9, 10, 11, 12 and gauge theories 13, 14, 15, 16, 17, 18 have been much studied in their own right. Intriguing phenomena occur, in particular there are important distinctions between planar and nonplanar Feynman diagrams even in theories of a single scalar. Moreover nonplanar diagrams are automatically regulated by an effective UV-cutoff $\Lambda_{\text {eff }}^{2} \sim 1 /(p \theta)^{2}$, where $p$ is some combination of external momenta. This implies a non-analyticity in $\theta$, and an IR singularity $\propto 1 /(p \theta)^{2}$ is generated from integrating out the high momentum modes. This UV/IR-mixing has attracted quite some attention. Within quantum field theory it appears as a puzzling feature, but if we think in terms of string theory there is no natural distinction between UV and IR since high energy open string loop excitations are mapped via a modular transformation to low energy closed string ones.

It is thus quite natural to suspect that the Seiberg-Witten limit of string theory with a non-vanishing $F$-field can be extended beyond tree-level. In fact, by now the low energy behavior $\left(\alpha^{\prime} \rightarrow 0\right)$ of string amplitudes is well understood also at one loop-level and has provided a reliable and flexible tool for analyzing various aspects of very different field theories. For instance, string amplitudes or string-inspired techniques were used to evaluate one-loop QCD scattering amplitudes [19, 20] (see also References therein) and renormalization constants [21, 22, 23]; graviton scattering amplitudes were computed and their relation to gauge amplitudes explored [24]; progress was made towards the extension of the method to more than one loop [25, 26, 27, 28, 29], and to off-shell amplitudes [30]. String techniques also served to stimulate the development of new techniques in field theory, that preserve some of the nice features of the string formalism [31]. Basically, the flexibility of these techniques has its root in the fact that string theory has a twodimensional structure, describing the world-sheet dynamics beyond the usual space-time structure. If one can find a corner of string moduli space which at low energies yields the field theory under study, it is possible to use the string description to perform the calculations and thus exploit all the conformal theory features one has already studied for other problems. Thus, it is not really a surprise that field theory computations, which are largely independent of each other, appear to be related if viewed from the string theory point of view.

As already discussed above, Seiberg and Witten have pointed out a regime of string theory which at low energies is described by a non-commutative theory and thus in the spirit of the above papers it is natural to exploit string computations to derive field theory results. Specifically, we will show how the non-commutative parameter $\theta$ arises 
in the field theory limit of one-loop amplitudes of the simple open bosonic string. We would like to insist that string theory not only conceptually leads to the noncommutative quantum field theories, but it also represents a simplifying technique for the computation of perturbative amplitudes. As we will explicitly see, once we have computed the objects entering in the string master formula also in the presence of a non-vanishing $F$-field, the one-loop diagrams of different noncommutative quantum field theories can be obtained by following the calculations performed in the commutative case; in particular, we refer to [23, 26, 28, 29. A nice feature discussed in detail in those references is the existence of a one-to-one correspondence between Feynman diagrams and different corners of the integration region over the string parameters. It is worth to stress that this mapping is preserved in the non-commutative case and is identical to the one found for $\theta=0$.

In order to incorporate a non-vanishing $F$-field in one-loop string computations, the first non-trivial task is to obtain the conformal field theory propagator with $F \neq 0$ on a worldsheet with topology of the annulus, rather than simply a disk. This will be done in section 2. Starting from the boundary state formalism, we discuss the possible ambiguity for the open string Green function that exists in the literature and provide an unambiguous computation to fix its form. Once this Green function is known, one can apply the techniques developed in [23, 26, 28, 29] in order to extract quantum field theory Feynman diagrams from string loop amplitudes. By means of this formalism, we compute various one-loop amplitudes in noncommutative scalar $\phi^{3}$ and $\phi^{4}$ field theories. In all cases we show that string theory exactly reproduces the previously known results obtained from quantizing the noncommutative field theory action. We then study the 2-gluon amplitude in noncommutative gauge theories and determine the leading and subleading singularity in $\theta$. By exploiting the fact that in the field theory limit, string theory gives results in the background field method [23], we easily obtain the $\beta$-function for the noncommutative $U(1)$ gauge theory.

Note added: After completing this work and while the present paper was typed, two related papers [44, 45] appeared where also one-loop noncommutative field theory amplitudes are obtained from one-loop string theory amplitudes. However, the Green function of [44] differs from ours since the above mentioned ambiguity was differently resolved. In section 2 we will argue that to obtain the correct gluon two-point function imposes our choice for resolving the ambiguity.

\section{One-loop open string Green function in the presence of an $F$-field}

In this section we focus on the one-loop Green function of bosonic string theory and, in particular, we want to generalize the usual calculation to the case where a constant field 
$F=B-d A$ is present. In fact, once the explicit form of the Green function is known, it is possible to write in a compact form a generic string amplitude with an arbitrary number of legs. The situation is thus very different from the one in field theory, where each diagram represents an independent calculation and one has always to start from the very first building blocks, i.e. the Feynman rules. As we said, this simplification is possible because string calculations rely on the world-sheet structure, which is described by a two-dimensional theory, more than on the space-time structure. In fact, the $h$-loop bosonic string amplitude among $M$-tachyons with inflowing momenta $p_{1}, \ldots, p_{M}$ can be written as

$$
A_{M}^{(h)}\left(p_{1}, \ldots, p_{M}\right)=C_{h} \mathcal{N}^{M} \int[d m]_{h}^{M} \prod_{i<j}\left[\frac{\exp \left(\mathcal{G}_{r_{i}, r_{j}}^{(h)}\left(\rho_{i}, \rho_{j}\right)\right)}{\sqrt{V_{i}^{\prime}(0) V_{j}^{\prime}(0)}}\right]^{2 \alpha^{\prime} p_{i} \cdot p_{j}}
$$

Here $C_{h}$ and $\mathcal{N}$ are the normalization factors depending on the world-sheet topology and the string vertices respectively; their explicit form in terms of the dimensionless string coupling constant will be given later. The other building blocks of (2.1) have a clear geometrical interpretation: $\mathcal{G}_{r_{i}, r_{j}}^{(h)}$ is the correlator of two world-sheet bosons located at $\rho_{i}$ on the boundary labeled $r_{i}$, and at $\rho_{j}$ on the boundary $r_{j} ;[d m]_{h}^{M}$ is the measure of integration over the moduli space for an open Riemann surface with $h$ loops and $M$ punctures; $V_{i}^{\prime}(\rho)$ are $M$ projective transformations which define local coordinate systems around each puncture $\rho_{i}$. Here we do not give the explicit expressions of these quantities in general (see for instance [32]), but we want to stress that their definition depends only on the geometrical properties of the string world-sheet and in general on the twodimensional conformal theory living on it. From this point of view it is natural that different computations are much more related to each other than in the usual field theory approach.

Here we want to exploit the great flexibility of this technique in order to derive the one-loop Feynman diagrams of noncommutative field theories; from the string point of view noncommutativity is easily implemented: one changes the commutation relations of the open string modes [3] or, equivalently, the boundary conditions of the open string coordinates $X^{\mu}(\sigma, \tau)$. At the tree and one-loop level this modification basically only shows up in the Green function. This means that the definition of the field theory limit of the string master formula is not modified by the presence of $F$; in particular, the mapping between the corners of integration over the moduli and Feynman diagrams can be read from the calculations of the usual commutative case [23]. Since in our approach all the differences between commutative and noncommutative field theory are resumed in the string Green function, we want to derive here this key ingredient from first principle. 


\subsection{Boundary state formalism}

In [8], among other things, the tree level Green function in the presence of a constant $F$ field was derived by solving the defining differential equation $\partial_{\rho} \partial_{\bar{\rho}} \mathcal{G}\left(\rho, \rho^{\prime}\right)=2 \pi \alpha^{\prime} \delta\left(\rho, \rho^{\prime}\right)$ with the following boundary condition 7

$$
\partial_{\perp} \mathcal{G}_{\mu \nu}\left(\rho, \rho^{\prime}\right)-\left.\mathrm{i} F_{\mu}^{\sigma} \partial_{\|} \mathcal{G}_{\sigma \nu}\left(\rho, \rho^{\prime}\right)\right|_{\rho=\bar{\rho}}=0
$$

where $\partial_{\|}\left(\partial_{\perp}\right)$ is the derivative parallel (normal) to the world-sheet border. There is however an ambiguity in this approach: one can always add to a given solution a constant piece (i.e. independent of the punctures $\rho, \rho^{\prime}$ ) with arbitrary dependence on $F$ and on the annulus width and obtain another Green function which gives different results in the $\alpha^{\prime} \rightarrow 0$ limit. As we will see, these terms play a crucial role in the field theory limit of noncommutative amplitudes, so it is important to understand the actual form of the Green function appearing in the string master formula (2.1). In order to clarify this point, we derive the Green function in the boundary state formalism using a simple trick that reduces the actual calculation to the one encountered in the usual case $F=0$.

Tree level

Let us consider the correlation function of two closed string tachyons on a disk, from which one may extract the tree-level Green function $\mathcal{G}^{(0)}$ by simply looking at the term with explicit dependence on both punctures

$$
A_{2}^{(0)} \sim\left\langle 0\left|\mathrm{e}^{\mathrm{i} p_{1} \cdot X(\rho, \bar{\rho})} \mathrm{e}^{\mathrm{i} p_{2} \cdot X\left(\rho^{\prime}, \bar{\rho}^{\prime}\right)}\right| 0\right\rangle d^{2} \rho d^{2} \rho^{\prime}=\mathrm{e}^{p_{1} \mathcal{G}^{(0)}\left(\rho, \rho^{\prime}\right) p_{2}+p_{1} \mathcal{C}^{(0)}(\rho) p_{1}+p_{2} \mathcal{C}^{(0)}\left(\rho^{\prime}\right) p_{2}} d^{2} \rho d^{2} \rho^{\prime} .
$$

This same amplitude can be calculated in the boundary state formalism. In this approach one starts from a world-sheet with the topology of the sphere and thus the string coordinates $X^{\mu}$ depend on two independent sets of oscillators $\alpha_{n}^{\mu}$ and $\tilde{\alpha}_{n}^{\mu}$. Then one introduces in the amplitudes a coherent state $|B\rangle$ (see [35] and References therein) which basically identifies the left and the right sector of the closed strings with the appropriate boundary conditions and thus inserts a boundary on the string world-sheet

$$
A_{2}^{(0)} \sim\left\langle 0, \tilde{0}\left|\mathrm{~T}\left(\mathrm{e}^{\mathrm{i} p_{1} \cdot X(z, \bar{z})} \mathrm{e}^{\mathrm{i} p_{2} \cdot X\left(z^{\prime}, \bar{z}^{\prime}\right)}\right)\right| B(P)\right\rangle d^{2} z d^{2} z^{\prime},
$$

where $\mathrm{T}$ is the radial ordering. The $P$ in the boundary state $|B(P)\rangle$ is put there to emphasize that in order to sew to a boundary state to a given Riemann surface, it has to contain a closed string propagator $1 /\left(L_{0}+\tilde{L}_{0}-2\right)$ [33]. At tree-level its effect in the amplitudes is just to shift the positions of the external legs, so it does not modify the form of the Green functions. Notice that in (2.3) and (2.4) we have used two different ways to label the world-sheet coordinates. This is because the two approaches naturally give rise to different parameterizations of the string world-sheet. In (2.3) the poles of open string

\footnotetext{
${ }^{1}$ Notice that there is a factor of $i$ different from (1.3) due to a Wick rotation on the world-sheet.
} 
exchanges between the two vertex operators are manifest and the fundamental region is the upper half of the complex plane. On the other hand, the boundary state calculation is written in the "closed string channel" and the world-sheet is mapped inside the disk of unit radius.

The advantage of the boundary state formulation is that it is very simple to introduce a constant $F$-field; in fact, one only needs to slightly modify the identification brought by the boundary state [34, 36] (here we use the convention of [36] in the particular case where there are no Dirichlet directions)

$$
\left[(1+F)_{\nu}^{\mu} \alpha_{n}^{\nu}+(1-F)_{\nu}^{\mu} \tilde{\alpha}_{-n}^{\nu}\right]|B\rangle_{F}=0, \forall n>0
$$

From this identification it is easy to see that the part of the tachyon vertex depending on the non-zero modes $\tilde{\alpha}_{n}$ satisfies the following relation

$$
: \mathrm{e}^{\mathrm{i} \frac{p}{2} \cdot \tilde{X}^{\prime}(\bar{z})}:|B\rangle_{F}=: \mathrm{e}^{\mathrm{i} \frac{p_{\mu}}{2}\left(\frac{1+F}{1-F}\right)_{\nu}^{\mu} X^{\prime \nu}\left(\frac{1}{\bar{z}}\right)}:|B\rangle_{F}
$$

where $X^{\prime}$ is the oscillator part of the string coordinate without the zero modes. In fact, since we only consider noncompact and Neumann directions in $|B\rangle$, both $p$ and $\tilde{p}$ vanish on the boundary; thus the zero mode contribution can be calculated separately and modifies only the $F$-independent part of $\mathcal{G}$. Using this identification repeatedly, one can reduce (2.4) to the the usual computation of the expectation value of four open string-like vertex operators and, in general, a $N$-point function of closed string on a disk is equivalent to a $2 N$-point amplitude among open strings. The only difference is that the former $\tilde{X}^{\mu}$ part of the vertices is evaluated in the unusual image point $1 / \bar{z}$ and its Lorentz index is contracted with the external momentum through a non-trivial matrix depending on $F$. After these considerations, it is easy to find the expression for the Green function $\mathcal{G}$ which depends on two different points $z$ and $z^{\prime}$

$$
\frac{1}{\alpha^{\prime}} \mathcal{G}_{\mu \nu}^{(0)}\left(z, z^{\prime}\right)=\delta_{\mu \nu} \ln \left|z-z^{\prime}\right|+\frac{1}{2}\left(\frac{1-F}{1+F}\right){ }_{\mu \nu} \ln \left(1-z \bar{z}^{\prime}\right)+\frac{1}{2}\left(\frac{1+F}{1-F}\right)_{\mu \nu} \ln \left(1-z^{\prime} \bar{z}\right) .
$$

Here the antisymmetric nature of the field $F$ has been used to rewrite the final result in the standard form where the index $\mu$ always precedes $\nu$. It is clear that $\mathcal{G}$ is symmetric under the simultaneous exchange $z \leftrightarrow z^{\prime}$ and $\mu \leftrightarrow \nu$.

Note that the above result is written in the $z$-coordinates, the parameterization chosen by the boundary state calculation. In order to do the comparison with the one of [\&], it is necessary to perform a conformal transformation $z=-(\rho-i) /(\rho+i)$ and rewrite the Green functions in terms of the $\rho$-coordinates. A small subtlety in this mapping comes from the fact that the Green function in (2.7) is not a scalar under conformal transformations. The simplest thing to do is to transform the scalar combination $\left(A_{2}^{(0)} d^{2} z d^{2} z^{\prime}\right)$ to the $\rho$ parameterization and read off $\mathcal{G}^{(0)}\left(\rho, \rho^{\prime}\right)$ from there. One finds indeed the same result (i.e. Eq. (2.15) of [8]). 
A couple of remarks are in order. Notice that the tree-level Green function in the zcoordinates cannot satisfy a boundary condition similar to the one in (2.2). This peculiarity has already been stressed in [8] where it was pointed out that the condition (2.2) may be in contradiction with the equation of motion because Gauss theorem requires that the sum of all boundary integrals is equal to $2 \pi \alpha^{\prime}$. This is indeed the case in the $z$ coordinate and the boundary condition is modified as

$$
\partial_{\perp} \mathcal{G}_{\mu \nu}\left(z, z^{\prime}\right)-\left.\mathrm{i} F_{\mu}{ }^{\sigma} \partial_{\|} \mathcal{G}_{\sigma \nu}\left(z, z^{\prime}\right)\right|_{|z|=1}=\alpha^{\prime}
$$

As already anticipated in the amplitudes among closed string vertices on a disk, one also gets a contribution $\mathcal{C}^{(0)}$ depending on a single point: this comes from the contraction of the former left and right moving part of the same vertex, since the oscillators of these two parts are now identified by the presence of a boundary (2.5)

$$
\frac{1}{\alpha^{\prime}} \mathcal{C}_{\mu \nu}^{(0)}(z)=\frac{1}{4}\left(\frac{1-F}{1+F}\right)_{\mu \nu} \ln \left(1-|z|^{2}\right)+\frac{1}{4}\left(\frac{1+F}{1-F}\right)_{\mu \nu} \ln \left(1-|z|^{2}\right)
$$

One loop

The same approach easily generalizes to one-loop, where now two boundary states must be inserted. Since the noncommutativity we are interested in at present is related to the global $U(1)$, we choose the boundary states to each enforce the same identification on the oscillators sets of closed strings,

$$
A_{2}^{(1)} \sim{ }_{F}\left\langle B(P)\left|\mathrm{T}\left(\mathrm{e}^{\mathrm{i} k_{1} \cdot X(z, \bar{z})} \mathrm{e}^{\mathrm{i} k_{2} \cdot X\left(z^{\prime}, \bar{z}^{\prime}\right)}\right)\right| B(P)\right\rangle_{F} d^{2} z d^{2} z^{\prime} .
$$

Again, one can use (2.6) in order to eliminate the dependence of the two vertices on the right moving oscillators, so that the scalar product $\langle B \mid B\rangle$ in this sector transforms into a trace over the remaining left moving $\alpha_{n}$ 's. The final evaluation of the trace is most easily performed by using coherent states and canonical forms [32]; let us here report and comment on the three basic pieces of the result. First the measure. The two propagators $P$ in (2.10) combine and give rise to the usual $q^{2 L_{0}}$ factor present in one-loop amplitudes. It is clear from this point of view that the contribution to (2.10) not appearing in the exponent depends trivially on $F$ only through the normalization of the boundary state [36]. This part is absorbed in the definition of the measure (the relation between $[d \mu]$ and $[d m]$ is given below) which thus becomes

$$
[d \mu]_{h=1}^{M=2}=\operatorname{det}(1+F)\left[\frac{d q}{q^{3}} d^{2} z d^{2} z^{\prime} \prod_{n=1}^{\infty}\left(1-q^{2 n}\right)^{2-d}\right] .
$$

Notice here that there is no $(\ln q)^{d / 2}$ in $[d \mu]$ since we did not have to perform any Gaussian integral in the closed string channel. Next, by looking at the exponent of the result (2.10), 
one can extract the one-loop Green function

$$
\begin{aligned}
\frac{1}{\alpha^{\prime}} \mathcal{G}_{\mu \nu}^{(1)}\left(z, z^{\prime}\right) & =\delta_{\mu \nu}\left[\ln \left|z-z^{\prime}\right|+\ln \prod_{n=1}^{\infty} \frac{\left|1-q^{2 n} \frac{z^{\prime}}{z}\right|\left|1-q^{2 n} \frac{z}{z^{\prime}}\right|}{\left(1-q^{2 n}\right)^{2}}\right] \\
& +\frac{1}{2}\left(\frac{1-F}{1+F}\right)_{\mu \nu}\left[\ln \left(1-z \bar{z}^{\prime}\right)+\ln \prod_{n=1}^{\infty} \frac{\left(1-q^{2 n} z \bar{z}^{\prime}\right)\left(1-\frac{q^{2 n}}{z \bar{z}^{\prime}}\right)}{\left(1-q^{2 n}\right)^{2}}\right] \\
& +\frac{1}{2}\left(\frac{1+F}{1-F}\right)_{\mu \nu}\left[\ln \left(1-z^{\prime} \bar{z}\right)+\ln \prod_{n=1}^{\infty} \frac{\left(1-q^{2 n} z^{\prime} \bar{z}\right)\left(1-\frac{q^{2 n}}{z^{\prime} \bar{z}}\right)}{\left(1-q^{2 n}\right)^{2}}\right] .
\end{aligned}
$$

Finally as in the tree-level case, the full amplitude $A_{2}^{(1)}$ contains also contractions between the left and the right part of a single vertex which are encoded in the following $\mathcal{C}^{(1)}$

$$
\begin{aligned}
\frac{1}{\alpha^{\prime}} \mathcal{C}_{\mu \nu}^{(1)}(z) & =\frac{1}{4}\left(\frac{1-F}{1+F}\right)_{\mu \nu}\left[\ln \left(1-|z|^{2}\right)+\ln \prod_{n=1}^{\infty} \frac{\left(1-q^{2 n}|z|^{2}\right)\left(1-\frac{q^{2 n}}{|z|^{2}}\right)}{\left(1-q^{2 n}\right)^{2}}\right] \\
& +\frac{1}{4}\left(\frac{1+F}{1-F}\right)_{\mu \nu}\left[\ln \left(1-|z|^{2}\right)+\ln \prod_{n=1}^{\infty} \frac{\left(1-q^{2 n}|z|^{2}\right)\left(1-\frac{q^{2 n}}{|z|^{2}}\right)}{\left(1-q^{2 n}\right)^{2}}\right] .
\end{aligned}
$$

We note that (2.12) is exactly the Green function obtained in [8] (Eq. (3.6) there), which satisfies a certain particular form of boundary condition. We remark that the result (2.12) are written in the "closed string channel" and the natural modular parameter $\ln q=\mathrm{i} \pi \tau_{c}$ is related to the length of the surface viewed as a cylinder. In this case a fundamental region for the string world-sheet is the annulus with inner radius $q$ and outer radius 1 . The result of [8] is obtained by setting $q=a / b$ and rescaling the coordinate $z \rightarrow z / b$.

In order to extract from the string amplitude the contribution of the "open string channel", where the world-sheet degenerates into a circle, one has to perform a modular transformation on both the Green function and the measure. In particular, at one-loop level the relation between $z$ and $\rho$-coordinates is [37]

$$
z=\mathrm{e}^{2 \pi \mathrm{i} \frac{\ln \rho}{\ln k}}, \quad \ln q=\frac{2 \pi^{2}}{\ln k} .
$$

Notice that this identification fixes the cut of the log function in the complex $\rho$ plane. In fact we want that the segment $(-1,-k)$ of the negative real axis is mapped by (2.14) on the inner border of the $z$-parameterization; thus we take 2

$$
\ln \rho=\ln |\rho|+i \arg \rho, \quad-\pi \leq \arg \rho<\pi
$$

\footnotetext{
${ }^{2}$ Of course, insisting on $-\pi \leq \arg \rho<\pi$ makes the logarithm a single valued function at the expense of continuity.
} 
However, before explicitly performing the modular transformation (2.14) on the various building blocks of the string amplitude, we want to make two important remarks about the Green function (2.12).

First as one can see from (2.1), the string amplitude does not contain simply $\mathcal{G}$, but its combination with the derivatives of the local coordinates around each punctures $V^{\prime}(0)$ [32], and this combination has conformal weight zero. Usually the $V^{\prime}(0)$ dependence drops out on-shell, since the factor coming from the exponent cancels against the one present in the definition of the the measure [26]

$$
[d m]_{1}^{M}=[d \mu]_{1}^{M} \prod_{i=1}^{m} 1 / V_{i}^{\prime}(0) .
$$

However, in order to exploit the off-shell continuation of the string results which is possible in the field theory limit [23, 26], it is more useful not to perform this simplification. Thus we use in the amplitudes the measure $[d m]$ instead of the one of (2.11), and a shifted Green function

$$
\mathbf{G}_{\mu \nu}^{(1)}\left(z, z^{\prime}\right)=\mathcal{G}_{\mu \nu}^{(1)}\left(z, z^{\prime}\right)-\frac{\alpha^{\prime}}{2} \delta_{\mu \nu}\left(\ln \left|V_{z}^{\prime}(0)\right|+\ln \left|V_{z^{\prime}}^{\prime}(0)\right|\right)=\mathcal{G}_{\mu \nu}^{(1)}\left(z, z^{\prime}\right)-\frac{\alpha^{\prime}}{2} \delta_{\mu \nu} \ln \left|z z^{\prime}\right| .
$$

Here, as in [23], we have related the derivative of the local coordinate to the one-loop Abelian differential $\omega$ since this is the only well defined object on the annulus having conformal dimension 1 . In particular we have $V_{i}^{\prime}(0)=z_{i}$.

The second remark is related to an ambiguity in the determination of $\mathcal{G}$ from the boundary state approach. In fact, as we have already seen, the computation of the amplitude (2.10) always gives a combination of $\mathcal{G}$ and $\mathcal{C}$. Thus, by exploiting momentum conservation, it is possible to shift terms of a particular form between $\mathcal{G}$ and $\mathcal{C}$. In particular one can extract from the closed string interaction on the annulus a different definition for $\mathcal{G}$ and $\mathcal{C}$ which is still compatible with the final result of 2.10

$$
\begin{aligned}
G_{\mu \nu}^{(1)}\left(z, z^{\prime}\right) & =\mathbf{G}_{\mu \nu}^{(1)}\left(z, z^{\prime}\right)+M_{\mu \nu}\left(z, z^{\prime}\right) \\
C_{\mu \nu}^{(1)}(z) & =\mathcal{C}_{\mu \nu}^{(1)}(z)-N_{\mu \nu}(z)
\end{aligned}
$$

The general form of this ambiguity is the addition to $\mathbf{G}_{\mu \nu}$ of a term $M_{\mu \nu}\left(z, z^{\prime}\right)$ with $M$ satisfying the Laplace equation, as well as $M_{\mu \nu}\left(z, z^{\prime}\right)=M_{\nu \mu}\left(z^{\prime}, z\right)$ in order to preserve the exchange symmetry discussed after (2.7). Moreover, in order to ensure that a shift of the form (2.18), (2.19) does not change the string amplitude for all mass levels, one also needs the following properties $M_{\mu \nu}\left(z, z^{\prime}\right)=-M_{\nu \mu}\left(z, z^{\prime}\right), \partial_{z} \partial_{z^{\prime}} M_{\mu \nu}=0$. Thus $M$ and $N$ are related by $M_{\mu \nu}\left(z, z^{\prime}\right)=N_{\mu \nu}(z)-N_{\mu \nu}\left(z^{\prime}\right)$. This freedom in the definition of the Green function also appears in the calculation of [8] where $\mathcal{G}$ is derived by solving the Laplace equation on the world-sheet. In fact, as in the tree level case, also here it is not possible to strictly impose on the Green function the same boundary condition imposed on the 
string coordinates (1.3); and hence there is a certain degree of freedom in the choice of what constraint is satisfied by $G$. Indeed, the shift in (2.18) corresponds simply to a redefinition of the boundary condition of the Green function which leaves unmodified the value of the integral $\oint \partial_{\perp} G d s=2 \pi \alpha^{\prime}$ that is fixed by Gauss' theorem. We stress that $G$ and $C$ give the same results as those obtained with $\mathbf{G}$ and $\mathcal{C}$ when they are used in the contraction of closed string fields $X(z)$ (where thus $z$ must be a point not located on the boundary of the surface). However, when one wants to restrict the Green function to the boundary in order to calculate open string amplitudes, the two Green functions may give different results.

For the case of scalar amplitudes, we note that the kind of shift in (2.18) does not modify the amplitude at all, as it is antisymmetric in $\mu$ and $\nu$ and the Green function is contracted only with external momenta. Therefore the new contributions due to the additional term sum up to zero using momentum conservation. Thus, the two Green functions $G^{(1)}$ and $\mathcal{G}^{(1)}$ actually give the same result for tachyon amplitudes. However this is not the case for gluon amplitudes as the string master formula will involve derivatives of the punctures, see (3.26) below. Using this in the next subsection, we will find that the correct Green function is given by (2.18).

\subsection{One-loop open string Green function with $F \neq 0$}

We begin by evaluating the Green function $\mathbf{G}_{\mu \nu}^{(1)}\left(z, z^{\prime}\right)$ of eq. (2.17), as obtained from the boundary state approach, when the arguments take values on any of the two boundaries. Then we will argue that we need to exploit the above-mentioned ambiguity and shift $\mathbf{G}_{\mu \nu}^{(1)}\left(z, z^{\prime}\right)$ to $G_{\mu \nu}^{(1)}\left(z, z^{\prime}\right)$ as in $(2.18)$ in order to correctly reproduce the gluon two-point amplitude in the noncommutative field theory limit.

First, we rewrite $\mathbf{G}_{\mu \nu}^{(1)}\left(z, z^{\prime}\right)$ in the following form, splitting it into its symmetric and antisymmetric part (in $\mu, \nu$ ):

$$
\frac{1}{\alpha^{\prime}} \mathbf{G}_{\mu \nu}^{(1)}\left(z, z^{\prime}\right)=\delta_{\mu \nu} \mathcal{I}+\left(\frac{1+F^{2}}{1-F^{2}}\right)_{\mu \nu} \mathcal{J}-\left(\frac{F}{1-F^{2}}\right)_{\mu \nu} \mathcal{K} \equiv \mathcal{S}+\mathcal{A}
$$

where

$$
\begin{aligned}
\mathcal{I} & =\ln \left|\sqrt{z / z^{\prime}}-\sqrt{z^{\prime} / z}\right|+\ln \prod_{n=1}^{\infty} \frac{\left|1-q^{2 n} \frac{z^{\prime}}{z}\right|\left|1-q^{2 n} \frac{z}{z^{\prime}}\right|}{\left(1-q^{2 n}\right)^{2}} \\
\mathcal{J} & =\ln \left|1-z \bar{z}^{\prime}\right|+\ln \prod_{n=1}^{\infty} \frac{\left|1-q^{2 n} z \bar{z}^{\prime}\right|\left|1-\frac{q^{2 n}}{z \bar{z}^{\prime}}\right|}{\left(1-q^{2 n}\right)^{2}} \\
\mathcal{K} & =\ln \left(\frac{1-z \bar{z}^{\prime}}{1-z^{\prime} \bar{z}}\right)+\ln \prod_{n=1}^{\infty} \frac{\left(1-q^{2 n} z \bar{z}^{\prime}\right)\left(1-\frac{q^{2 n}}{z \bar{z}^{\prime}}\right)}{\left(1-q^{2 n} z^{\prime} \bar{z}\right)\left(1-\frac{q^{2 n}}{z^{\prime} \bar{z}}\right)} .
\end{aligned}
$$


It is easy to see that $\mathbf{G}^{(1)}\left(z, z^{\prime}\right)$ is invariant under $z \rightarrow q / z, z^{\prime} \rightarrow q / z^{\prime}$ (equivalently $\left.\rho \rightarrow-k / \rho, \rho^{\prime} \rightarrow-k / \rho^{\prime}\right)$ which maps the outer boundary of the annulus to the inner one and vice versa. Note also that $\mathbf{G}^{(1)}\left(z, z^{\prime}\right)$ is single valued on the annulus.

As we have already said, the field theory limit of string amplitudes is more easily performed in the Schoktty representation of the annulus, since there the open string contributions are manifest. To go to the $\rho$ coordinate, the conformal transformation (2.14) implies the following transformation

$$
\begin{aligned}
& \ln \left(\sqrt{z / z^{\prime}}-\sqrt{z^{\prime} / z}\right)+\ln \prod_{n=1}^{\infty} \frac{\left(1-q^{2 n} \frac{z^{\prime}}{z}\right)\left(1-q^{2 n} \frac{z}{z^{\prime}}\right)}{\left(1-q^{2 n}\right)^{2}}+\ln \left(\frac{-\pi}{\ln q}\right) \\
= & \frac{\ln ^{2} \rho / \rho^{\prime}}{2 \ln k}+\ln \left(\sqrt{\rho / \rho^{\prime}}-\sqrt{\rho^{\prime} / \rho}\right)+\ln \prod_{n=1}^{\infty} \frac{\left(1-k^{n} \frac{\rho}{\rho^{\prime}}\right)\left(1-k^{n} \frac{\rho^{\prime}}{\rho}\right)}{\left(1-k^{n}\right)^{2}} .
\end{aligned}
$$

Let us discuss the symmetric part of $\mathbf{G}^{(1)}$ first. The non-planar open string Green function has one argument on each boundary, i.e. $|z|=1$ and $\left|z^{\prime}\right|=q$ corresponding to $\rho>0$ and $\rho^{\prime}<0$, or vice versa. For the planar case, a priori, one has to distinguish the case where both arguments are on the outer boundary $\left(|z|=\left|z^{\prime}\right|=1\right.$ corresponding to $\left.\rho, \rho^{\prime}>0\right)$ from the case where both arguments are on the inner boundary $\left(|z|=\left|z^{\prime}\right|=q\right.$ corresponding to $\left.\rho, \rho^{\prime}<0\right)$. It is easy to see that for both planar cases $\left(\rho / \rho^{\prime}>0\right)$

$$
\mathcal{I}=I_{0}-\ln \left(\frac{-\pi}{\ln q}\right)
$$

where we have separated the $\ln (\ln q)$ term, which will eventually combine with the modular transformation of the measure, from the usual Green function $I_{0}$

$$
I_{0}\left(\rho, \rho^{\prime}\right)=\frac{\ln ^{2}\left(\rho / \rho^{\prime}\right)}{2 \ln k}+\ln \left|\sqrt{\frac{\rho}{\rho^{\prime}}}-\sqrt{\frac{\rho^{\prime}}{\rho}}\right|+\ln \prod_{n=1}^{\infty}\left|\frac{\left(1-k^{n} \frac{\rho}{\rho^{\prime}}\right)\left(1-k^{n} \frac{\rho^{\prime}}{\rho}\right)}{\left(1-k^{n}\right)^{2}}\right| .
$$

For the nonplanar case we have $\rho / \rho^{\prime}<0$ and taking into account (2.15),

$$
\ln \rho / \rho^{\prime}=\ln \left|\rho / \rho^{\prime}\right|-i \pi
$$

so that $\left|\mathrm{e}^{\frac{\ln ^{2} \rho / \rho^{\prime}}{2 \ln k}}\right|=\mathrm{e}^{\frac{1}{2 \ln k}\left(\ln ^{2}\left|\rho / \rho^{\prime}\right|-\pi^{2}\right)}$ and hence

$$
\mathcal{I}=-\frac{\pi^{2}}{2 \ln k}+I_{0}-\ln \left(\frac{-\pi}{\ln q}\right)
$$

with $I_{0}$ given by

$$
I_{0}\left(\rho, \rho^{\prime}\right)=\frac{\ln ^{2}\left|\rho / \rho^{\prime}\right|}{2 \ln k}+\ln \left(\sqrt{\left|\frac{\rho}{\rho^{\prime}}\right|}+\sqrt{\left|\frac{\rho^{\prime}}{\rho}\right|}\right)+\ln \prod_{n=1}^{\infty}\left|\frac{\left(1+k^{n}\left|\rho / \rho^{\prime}\right|\right)\left(1+k^{n}\left|\rho^{\prime} / \rho\right|\right)}{\left(1-k^{n}\right)^{2}}\right| .
$$


Next one has to transform the other piece $\mathcal{J}$ to the $\rho$ coordinate, and then restrict the values of $\rho$ and $\rho^{\prime}$ to the appropriate boundaries. While this is straightforward, it is simpler to remark, that when first restricting $z$ and $z^{\prime}$ to the boundaries one easily sees that

$$
\mathcal{J}=\mathcal{I}+ \begin{cases}0, & \text { planar (outer or inner) } \\ \frac{1}{2} \ln q, & \text { nonplanar. }\end{cases}
$$

As a result, we obtain for the symmetric part

$$
\frac{\left(1-F^{2}\right)}{2} \mathcal{S}=I_{0}-\ln \left(\frac{-\pi}{\ln q}\right)+ \begin{cases}0, & \text { planar (outer or inner) } \\ \frac{\theta^{2}}{8 \alpha^{\prime 2} \ln k}, & \text { nonplanar }\end{cases}
$$

where we have identified

$$
\theta^{\mu \nu}=2 \pi \alpha^{\prime} F^{\mu \nu}
$$

As mentioned before, the noncommutative field limit is defined by $\alpha^{\prime} \rightarrow 0$ with $\alpha^{\prime} F$ fixed and hence $\theta$ is a fixed quantity in field theory.

Now we turn to the antisymmetric part $\mathcal{A}$. We will transform this to the $\rho$ coordinates and then evaluate it on the boundaries. Since $z \bar{z}^{\prime}=\exp \left(\frac{2 \pi i}{\ln k} \ln \frac{\rho}{\bar{\rho}^{\prime}}\right)$, one has to be careful to correctly take into account the cut of the logarithm. For the planar case with both $z, z^{\prime}$ on the outer boundary $\left(\rho, \rho^{\prime}>0\right)$ there is no subtlety. With the choice of the cut $(2.15)$, the non-planar cases also present no difficulties as we can always take $-\pi \leq \arg \left(\rho / \bar{\rho}^{\prime}\right)<0$, and the value of the Green function on the boundary is just obtained by analytically continuing the Green function from the interior of the $\rho$-domain. However, to get the planar Green function with both $z$ and $z^{\prime}$ on the inner boundary, we cannot analytically continue the resulting expression without encountering the cut, since now the argument of $\rho / \bar{\rho}^{\prime}$ would have to go from 0 to $-2 \pi$. The simplest and safest way to get the Green function for this case is to observe that $G_{\mu \nu}\left(\rho, \rho^{\prime}\right)$ must always be periodic under $\rho \rightarrow k \rho, \rho^{\prime} \rightarrow k \rho^{\prime}$, as well as under $\rho \rightarrow-k / \rho, \rho^{\prime} \rightarrow-k / \rho^{\prime}$. The second transformation exchanges the outer and inner boundary, so that the planar Green function on the inner boundary equals the one on the outer boundary. Thus, rather than insisting on analytic continuation which physically is irrelevant here, since only the Green functions on the boundaries are relevant, we insist on the physical equivalence of the two boundaries.

As already pointed out, $\mathcal{A}$ has an ambiguity that cannot be fixed in the boundary state computation. This ambiguity does not affect the scalar amplitudes as it gives a vanishing contribution when substituted into the string master formula (2.1). However there is an important difference in the gluon amplitude. Indeed, the gluon master formula (3.26) contains terms that depend on the derivatives with respect to the punctures and there is only one form of the Green function that can give the correct field theory result. The ambiguity can be most easily fixed by looking at the gluon 2-point function. We recall from field theory that the planar gluon 2-point function is independent of $\theta$. It is easy to 
see that if $|z|=\left|z^{\prime}\right|=1, \mathcal{K}^{\mathrm{P}}=\ln \left(-z / z^{\prime}\right)$ and using this in the master formula would lead to a $\theta$-dependence of the planar 2-gluon amplitude. Indeed on the boundary we have

$$
\mathcal{K}^{\mathrm{P}}= \pm \ln \left(\frac{-z}{z^{\prime}}\right) \quad, \quad \mathcal{K}^{\mathrm{NP}}=0
$$

where $+(-)$ refers to the outer (inner) boundary. However, the field theory result can be reproduced if $\mathcal{K}$ is shifted as follows

$$
\mathcal{K}^{\mathrm{P}}=-\mathrm{i} \pi \epsilon\left(\rho-\rho^{\prime}\right) \quad, \quad \mathcal{K}^{\mathrm{NP}}=\mp \frac{2 \pi \mathrm{i}}{\ln k} \ln \left|\rho \rho^{\prime}\right|,
$$

which amounts to choose the $N_{\mu \nu}(z)$ introduced in the previous section as $\pm \alpha^{\prime} F /(1-$ $\left.F^{2}\right) \ln (z)$. Thus, the final result is

$$
\frac{\left(1-F^{2}\right)}{2} \mathcal{A}= \begin{cases}-\frac{i \theta}{4 \alpha^{\prime}} \epsilon\left(\rho-\rho^{\prime}\right), & \text { planar (outer or inner) } \\ \pm \frac{i \theta}{2 \alpha^{\prime} \ln k} \ln \left|\rho \rho^{\prime}\right|, & \text { nonplanar }\end{cases}
$$

where $\epsilon(\rho)$ is the step function that is 1 or -1 for positive or negative $\rho$. Note that this open string Green function is to be taken only on the boundaries (real $\rho, \rho^{\prime}$ ). Note also that $G_{\mu \nu}^{(1)}\left(\rho, \rho^{\prime}\right)$ is symmetric under the exchange of the two particles:

$$
G_{\mu \nu}^{(1)}\left(\rho, \rho^{\prime}\right)=G_{\nu \mu}^{(1)}\left(\rho^{\prime}, \rho\right)
$$

We see that the only modification in the planar case is a step function and it gives rise to the usual phase factor of 38$]^{3}$.

Before substituting (2.29) and (2.33) into the string master formula for the open string amplitude, we note that one has to scale them by a factor of $\left(1-F^{2}\right) / 2$ first. The factor of 2 is simply because a different normalization for the Green function was adopted for the boundary state formalism and the open string amplitude (for instance, because of (2.12), the exponent of (2.3) is proportional to $\alpha^{\prime} p^{2}$, while in (2.1) a factor of $2 \alpha^{\prime}$ is present). As for the scaling $\left(1-F^{2}\right)$, it is needed when one passes from the closed to the open string amplitudes. The reason is simple. Let's consider the case of tachyon states whose vertex operator is $\mathrm{e}^{\mathrm{i} p \cdot X}$ when $\theta=0$. As usual one may read the mass of the ground state described by the above vertex by simply looking at the Virasoro constraint $L_{0}-1=0$. When the non-commutative parameter is turned on, the commutation relations for the modes become [3, 7],

$$
\begin{gathered}
{\left[a_{n}^{\mu}, x_{0}^{\nu}\right]=\left[a_{n}^{\mu}, p_{0}^{\nu}\right]=\left[p_{0}^{\mu}, p_{0}^{\nu}\right]=0} \\
{\left[a_{m}^{\mu}, a_{n}^{\nu}\right]=2 \alpha^{\prime} m M^{-1 \mu \nu} \delta_{m+n} \quad\left[x_{0}^{\mu}, p_{0}^{\nu}\right]=\mathrm{i} 2 \alpha^{\prime} M^{-1 \mu \nu}} \\
{\left[x_{0}^{\mu}, x_{0}^{\nu}\right]=\mathrm{i} 2 \pi \alpha^{\prime}\left(M^{-1} F\right)^{\mu \nu}}
\end{gathered}
$$

\footnotetext{
${ }^{3}$ Note that putting the labels $r$ in increasing order in clockwise or anticlockwise direction is a matter of convention, changing $\theta$ to $-\theta$ and hence in individual Feynman diagrams. However the total amplitude is always an even function of $\theta$. We will take the convention of clockwise ordering in this paper.
} 
where $M=1-F^{2}$ and

$$
X^{\mu}=x_{0}^{\mu}+\left(p_{0}^{\mu} \tau-p_{0}^{\nu} F_{\nu}{ }^{\mu} \sigma\right)+\sum_{n \neq 0} \frac{\mathrm{e}^{-\mathrm{i} n \tau}}{n}\left(\mathrm{i} a_{n}^{\mu} \cos n \sigma-a_{n}^{\nu} F_{\nu}{ }^{\mu} \sin n \sigma\right),
$$

is the mode expansion for the open string coordinates. The change in (2.35) and (2.36) is gentle since it is simply an $F$ dependent rescaling [8, 39],

$$
\hat{x}_{0}^{\mu}=x_{0}^{\nu}(1-F)_{\nu}^{\mu}, \quad \hat{p}_{0}^{\mu}=p_{0}^{\nu}(1-F)_{\nu}^{\mu}, \quad \hat{a}_{n}^{\mu}=a_{n}^{\nu}(1-F)_{\nu}{ }^{\mu} .
$$

In terms of these operators, the commutation relations (2.35), (2.36) take the standard form, with the $F$-dependence concentrated on

$$
\left[\hat{x}_{0}^{\mu}, \hat{x}_{0}^{\nu}\right]=2 \pi \mathrm{i} \alpha^{\prime} F^{\mu \nu} .
$$

However since $\hat{x}_{0}$ does not show up in $L_{0}$, the computation of the mass parallels the calculation for $\theta=0$ and is found to be $F$ dependent. On the other hand, in the field theories we want to reproduce, the presence of the noncommutative parameter has no effect on the quadratic part of the Lagrangian, so the mass of the fields do not depend on $\theta$. In order to reproduce this feature in the string amplitude, we rescale quantum numbers like the external momenta and polarizations by an appropriate factor $p^{\mu} \rightarrow$ $(p \cdot(1-F))^{\mu}$. Notice, as a check, that this rescaling exactly absorbs the overall $F$ dependent normalization of the measure found in the boundary state calculation (2.11). Equivalently one can introduce the hatted variables as in the previous subsection. With this, the mass of the tachyon takes the usual value $-1 / \alpha^{\prime}$. We remark that this step of rescaling the modes by $1-F$ is equivalent to using a vertex operator $V=e^{i p \cdot \hat{X}}$ with the rescaled open string coordinate $\hat{X}=X(1-F)$.

As we have mentioned before, we note that there is no $\ln q$ factor in the closed string amplitude just like the $\theta=0$ case, but there is a power of $(\ln k)^{-d / 2} \sim(\ln q)^{d / 2}$ in the open string amplitude. There are three sources that these $l n q$ factors can arise when passing from the closed string to the open string amplitude: from the modular transformation (2.22); from the measure of integration over the moduli; and from the partition function. All these factors are independent of $F$ and they combine to give the desired $l n k$ dependence of the open string amplitude.

Summarizing, the open string Green function with constant F-field in the Schottky representation of the annulus is given by

$$
G_{P}^{\mu \nu}\left(\rho, \rho^{\prime}\right)=I_{0} \delta^{\mu \nu}-\frac{\mathrm{i} \theta^{\mu \nu}}{4 \alpha^{\prime}} \epsilon\left(\rho-\rho^{\prime}\right)
$$

with

$$
I_{0}\left(\rho, \rho^{\prime}\right)=\frac{\ln ^{2}\left(\rho / \rho^{\prime}\right)}{2 \ln k}+\ln \left|\sqrt{\frac{\rho}{\rho^{\prime}}}-\sqrt{\frac{\rho^{\prime}}{\rho}}\right|+\ln \prod_{n=1}^{\infty}\left|\frac{\left(1-k^{n} \frac{\rho}{\rho^{\prime}}\right)\left(1-k^{n} \frac{\rho^{\prime}}{\rho}\right)}{\left(1-k^{n}\right)^{2}}\right|
$$


in the planar case; and the nonplanar Green function is

$$
G_{N P}^{\mu \nu}\left(\rho, \rho^{\prime}\right)=I_{0} \delta^{\mu \nu}+\frac{\left(\theta^{2}\right)^{\mu \nu}}{8 \alpha^{\prime 2}} \frac{1}{\ln k} \pm \frac{i \theta^{\mu \nu}}{2 \alpha^{\prime}} \frac{\ln \left|\rho \rho^{\prime}\right|}{\ln k}
$$

with

$$
I_{0}\left(\rho, \rho^{\prime}\right)=\frac{\ln ^{2}\left|\rho / \rho^{\prime}\right|}{2 \ln k}+\ln \left(\sqrt{\left|\frac{\rho}{\rho^{\prime}}\right|}+\sqrt{\mid \frac{\rho^{\prime}}{\rho}} \mid\right)+\ln \prod_{n=1}^{\infty}\left|\frac{\left(1+k^{n}\left|\rho / \rho^{\prime}\right|\right)\left(1+k^{n}\left|\rho^{\prime} / \rho\right|\right)}{\left(1-k^{n}\right)^{2}}\right| .
$$

The sign $+(-)$ in $(2.43)$ refers to the outer (inner) borders. Note that $G$ is still symmetric with respect to the exchange of particles

$$
G^{\mu \nu}\left(\rho, \rho^{\prime}\right)=G^{\nu \mu}\left(\rho^{\prime}, \rho\right) .
$$

Exactly these open string Green functions are obtained from the open string operator formalism [46].

\section{String amplitudes in the presence of a constant $F$-field and their field theory limits}

After this detailed discussion of the Green functions and the measure in the previous section, we now turn to the actual evaluation of the one-loop open string amplitudes. In section 3.1 we start our analysis by focusing on scalar interactions; this is the simplest example since the scalar amplitudes involve only the ground state of open bosonic string theory (that is the tachyon). In section 3.2, we study the Yang-Mills case.

As a general remark, we want to stress that string amplitudes yield the correct overall normalization of the various Feynman diagrams without having to calculate the combinatorial factors typical of field theory and this agreement holds also in the non-planar case 29]. It is natural then to expect that also the coefficient of noncommutative amplitudes are reproduced by the string master formula. We find that this is indeed the case without having to change the definition of $\mathcal{C}_{h}$ and $\mathcal{N}$. We have [23, 26, 33, 34, 29]

$$
C_{h}=\frac{1}{(2 \pi)^{d h}} g_{\mathrm{op}}{ }^{2 h-2} \frac{1}{\left(2 \alpha^{\prime}\right)^{d / 2}} \quad, \quad \mathcal{N}=\sqrt{2 r} g_{\mathrm{op}}\left(2 \alpha^{\prime}\right)^{\frac{d-2}{4}}
$$

where $g_{\mathrm{op}}$ is the open string coupling constant and $r$ is related to the normalization chosen for the Chan-Paton factor $\operatorname{Tr}\left(\lambda^{a} \lambda^{b}\right)=\frac{1}{r} \delta^{a b}$. Note, in particular, that the vertex normalization $\mathcal{N}$ is independent of the particular string state chosen [23] and will be used to derive both scalar and "photon" interactions. 


\subsection{One-loop amplitudes in scalar theories}

We are now in the position to derive from the master formula (2.1) a compact expression that will generate the noncommutative Feynman diagrams for scalar theoriest

$$
\begin{aligned}
A_{M}^{(1)}\left(p_{1}, \ldots, p_{M}\right) & =\frac{\left(\sqrt{2} g_{\mathrm{op}}\right)^{M}}{(4 \pi)^{d / 2}}\left(2 \alpha^{\prime}\right)^{(M d-2 M-2 d) / 4} \int_{0}^{1} \frac{d k}{k} \mathrm{e}^{\alpha^{\prime} m^{2} \ln k}\left(-\frac{\ln k}{2}\right)^{-d / 2} \\
& \times \int \frac{d \rho_{2}}{\rho_{2}} \cdots \int \frac{d \rho_{M}}{\rho_{M}} \exp \left[\sum_{i<j} 2 \alpha^{\prime} p_{i}^{\mu} p_{j}^{\nu} G_{\mu \nu}^{(\mathrm{E})}\left(\rho_{i}, \rho_{j}\right)\right] .
\end{aligned}
$$

The string projective invariance has been used to choose the fixed points of the single Schottky generator as $\eta=0$ and $\xi \rightarrow \infty$, and to fix $\rho_{1}=1$. Also, following [26], we have to neglect all $O(k)$ terms in the measure of the integration. In fact they will not be relevant to the field theory limit where we want to single out the contributions where only scalar particles ("tachyon") run in the loop. Notice also that the original string measure is quadratically divergent when $k \rightarrow 0$, which just signals the presence of a tachyon instability in the bosonic string. In the field theory limit, however, we want to deal with scalars of positive $m^{2}$, so we replace the tachyon mass $-\frac{1}{\alpha^{\prime}} \rightarrow m^{2}$ and $k^{-1} \rightarrow \mathrm{e}^{\alpha^{\prime} m^{2} \ln k}$. This may look strange at first sight, but it has a natural interpretation if one goes back to the old dual model [26] and is sufficient to reproduce correctly all scalar field theory diagrams.

In the same spirit one does not have to use the full Green function derived in the previous sections, but can use the effective $G^{(\mathrm{E})}$ where all positive powers of $k$, corresponding to higher string modes, have been dropped

$$
\begin{aligned}
& G_{\mu \nu \mathrm{P}}^{(\mathrm{E})}\left(\rho_{i}, \rho_{j}\right)=\delta_{\mu \nu}\left[\ln \left|\sqrt{\frac{\rho_{i}}{\rho_{j}}}-\sqrt{\frac{\rho_{j}}{\rho_{i}}}\right|+\frac{1}{2 \ln k} \ln ^{2} \frac{\rho_{i}}{\rho_{j}}\right]-\frac{\mathrm{i} \theta_{\mu \nu}}{4 \alpha^{\prime}} \epsilon\left(\rho_{i}-\rho_{j}\right) \\
& G_{\mu \nu \mathrm{NP}}^{(\mathrm{E})}\left(\rho_{i}, \rho_{j}\right)=\delta_{\mu \nu}\left[\ln \left(\sqrt{\frac{\left|\rho_{i}\right|}{\left|\rho_{j}\right|}}+\sqrt{\frac{\left|\rho_{j}\right|}{\left|\rho_{i}\right|}}\right)+\frac{1}{2 \ln k} \ln ^{2} \frac{\left|\rho_{i}\right|}{\left|\rho_{j}\right|}\right] \pm \frac{\mathrm{i} \theta_{\mu \nu}}{2 \alpha^{\prime}} \frac{\ln \left|\rho_{i} \rho_{j}\right|}{\ln k}+\frac{\left(\theta^{2}\right)_{\mu \nu}}{8\left(\alpha^{\prime}\right)^{2}} \frac{1}{\ln k},
\end{aligned}
$$

for the planar and non-planar case respectively.

As it is clear from the above expression, for $\theta=0$, the differences between the planar and nonplanar Green functions are just some absolute values and signs and it is easy to see that they lead to the same field theory integrand. This is no longer true when a non-zero $F$-field is turned on. In this case, the non-planar Green function is modified by some non-trivial terms which depend both on $\theta$ and on the moduli $\rho$ and $k$. As we will see, this

\footnotetext{
${ }^{4}$ Notice that the overall normalization, in terms of $g_{\text {op }}$ is different from the one of [29] because there the convention $r=2$ has been used. Here we focus on $U(1)$ interaction and thus is more natural to fix $r=1$.
} 
modification is enough to account for all the differences in noncommutative field theory between planar and non-planar diagrams.

$\phi^{3}$ in 6 dimensions

The first noncommutative field theory we want to reproduce has only $\phi^{3}$ interactions:

$$
S_{3}=\int \frac{1}{2} \partial_{\mu} \phi \partial^{\mu} \phi-\frac{1}{2} m^{2} \phi^{2}+\frac{1}{3 !} g_{3}(\phi * \phi * \phi) .
$$

By comparing the result for the simplest tree-level amplitude between string and field theory, one can fix the relation between $g_{\text {op }}$ and $g_{3}$

$$
g_{3}=2^{5 / 2} g_{\mathrm{op}}\left(2 \alpha^{\prime}\right)^{\frac{d-6}{4}} .
$$

Let us now turn to loop amplitudes. As a warm-up exercise we will first compute the one-loop two-point function in scalar $\phi^{3}$-theory from string theory. This example will show most of the necessary ingredients.

By writing (3.2) for the special case $M=2$, one gets

$$
A_{2}^{\mathrm{P}}\left(p_{1},-p_{1}\right)=\frac{\alpha^{\prime 2-d / 2}}{(4 \pi)^{d / 2}} \frac{g_{3}^{2}}{4} \int_{0}^{1} \frac{d k}{k} \mathrm{e}^{\alpha^{\prime} m^{2} \ln k}(-\ln k)^{-d / 2} \int_{k}^{1} \frac{d \rho_{2}}{\rho_{2}} \mathrm{e}^{\left[-2 \alpha^{\prime} p_{1}^{\mu} p_{1}^{\nu} G_{\mu \nu}^{\mathrm{P}}\left(1, \rho_{2}\right)\right]},
$$

since $\rho_{1}=1$ is fixed. The field theory limit $\left(\alpha^{\prime} \rightarrow 0\right)$ of the above expression has to be performed, as usual, by keeping fixed all quantities that have a meaning in the field theory. In particular the logarithmic divergences in the Green function are related to the dimensionfull Schwinger parameters via a factor of $\alpha^{\prime}$. For instance, one always associates $\ln k$ to the total length of the loop by taking

$$
\ln k=-\frac{T}{\alpha^{\prime}},
$$

thus $T$ has to be kept finite as $\alpha^{\prime} \rightarrow 0$. In this limit $k$ goes to zero exponentially which means shrinking the annulus to a one-loop Feynman graph. After this replacement the $\alpha^{\prime}$ dependence of (3.6) simplifies and the whole amplitude is just proportional to a single power of $\alpha^{\prime}$. This means that, in order to have a finite answer for our field theory limit, it is necessary to introduce one more Schwinger parameter: since we want to reproduce the irreducible diagram, we perform the $\alpha^{\prime} \rightarrow 0$ limit by keeping fixed also $t_{2}$

$$
\ln \rho_{2}=-\frac{t_{2}}{\alpha^{\prime}}
$$

and get

$$
A_{2}^{\mathrm{P}}\left(p_{1},-p_{1}\right)=\frac{g_{3}^{2}}{4} \frac{1}{(4 \pi)^{d / 2}} \int_{0}^{\infty} \frac{d T}{T^{d / 2}} \mathrm{e}^{-m^{2} T} \int_{0}^{T} d t_{2} \exp \left[-p_{1}^{2} t_{2}\left(1-\frac{t_{2}}{T}\right)\right],
$$


where we have taken the $\alpha^{\prime} \rightarrow 0$ limit, since no overall $\alpha^{\prime}$ factor remained. In this simple case, the $\theta$-term does not contribute since $p_{1}^{\mu} \theta_{\mu \nu} p_{1}^{\nu}=0$. (3.9) matches the standard field theory result written in the Schwinger parameterization, numerical factor included. Notice that the overall $\frac{1}{4}$ is half of the usual result determined by the calculation of the symmetry factor, because we have restricted ourself to the planar diagram.

Now we look at the nonplanar contribution to the 2-point one-loop open string amplitude. Then $\rho_{1}=1$ and $\rho_{2} \in[-1,-k]$, so (3.8) is replaced by

$$
\ln \left|\rho_{2}\right|=-\frac{t_{2}}{\alpha^{\prime}}
$$

Then, using the nonplanar Green function (2.43) yields

$$
A_{2}^{\mathrm{NP}}\left(p_{1},-p_{1}\right)=\frac{g_{3}^{2}}{4} \frac{1}{(4 \pi)^{d / 2}} \int_{0}^{\infty} \frac{d T}{T^{d / 2}} \mathrm{e}^{-m^{2} T} \int_{0}^{T} d t_{2} \exp \left[-p_{1}^{2} t_{2}\left(1-\frac{t_{2}}{T}\right)+p_{1}^{\mu} p_{1}^{\nu} \frac{\theta_{\mu \nu}^{2}}{4 T}\right] .
$$

Again, we are left with the standard Schwinger proper time integral, but with the additional factor

$$
\mathrm{e}^{\frac{1}{4 T} p_{1}^{\mu} \theta_{\mu \nu}^{2} p_{1}^{\nu}}=\mathrm{e}^{-\frac{1}{4 T} \tilde{p}_{1}^{2}} \quad \text { with } \quad \tilde{p}_{\mu}=\theta_{\mu \nu} p^{\nu} .
$$

As noted in [10, for $\tilde{p}^{2} \neq 0$ this serves as an effective UV cutoff and is at the origin of the UV/IR mixing. The amplitude (3.11) exactly coincides with the one obtained from a direct one-loop calculation in noncommutative $\phi^{3}$ field theory.

Let us now consider the irreducible part of the 3-point amplitude. In the noncommutative $\phi^{3}$ field theory, there are 2 planar and 6 nonplanar diagrams. The 2 planar ones correspond to the two different cyclic orderings of $p_{1}, p_{2}$ and $p_{3}$ and are each equal to one half of the standard commutative diagram times an overall factor $e^{-\frac{i}{2} p_{1}^{\mu} p_{2}^{\nu} \theta_{\mu \nu}}$ resp. $e^{+\frac{i}{2} p_{1}^{\mu} p_{2}^{\nu} \theta_{\mu \nu}}$. In string theory we have one planar diagram with $k<\rho_{2}, \rho_{3}<\rho_{1} \equiv 1$ which can be splitted into $\rho_{2}<\rho_{3}$ and $\rho_{2}>\rho_{3}$, leading to the 2 different planar noncommutative field theory diagrams as $\alpha^{\prime} \rightarrow 0$. We also have a nonplanar string amplitude where we have three choices of which $\rho$ is alone on one boundary, and for each such choice there are two possible orderings of the two $\rho$ 's which are not fixed. Again we will show how the noncommutative field theory one-loop amplitudes are obtained from the open string one-loop amplitude by studying a particular example. The other diagrams are obtained in a completely analogous manner. We will start with the nonplanar string loop having $\rho_{1}=1$ on one boundary and $\rho_{2}, \rho_{3} \in[-1,-k]$ on the other boundary. From the general formula (3.2) we get

$$
\begin{aligned}
& A_{3}^{\mathrm{NP}}\left(p_{1} ; p_{2}, p_{3}\right)=\frac{g_{3}^{3}}{8} \frac{\left(2 \alpha^{\prime}\right)^{3-d / 2}}{(4 \pi)^{d / 2}} \int_{0}^{1} \frac{d k}{k^{2}}(-\ln k)^{-d / 2} \int_{-1}^{-k} \frac{d \rho_{2}}{\rho_{2}} \int_{\rho_{2}}^{-k} \frac{d \rho_{3}}{\rho_{3}} \\
& \times \exp \left[2 \alpha^{\prime} p_{1}^{\mu} p_{2}^{\nu} G_{\mu \nu}^{\mathrm{NP}}\left(1, \rho_{2}\right)+2 \alpha^{\prime} p_{1}^{\mu} p_{3}^{\nu} G_{\mu \nu}^{\mathrm{NP}}\left(1, \rho_{3}\right)+2 \alpha^{\prime} p_{2}^{\mu} p_{3}^{\nu} G_{\mu \nu}^{\mathrm{P}}\left(\rho_{2}, \rho_{3}\right)\right] .
\end{aligned}
$$


Now let $\left(-1<\rho_{2}<\rho_{3}<-k\right)$

$$
\ln k=-\frac{T}{\alpha^{\prime}}, \quad \ln \left|\rho_{2}\right|=-\frac{t_{2}}{\alpha^{\prime}}, \quad \ln \left|\rho_{3}\right|=-\frac{t_{2}+t_{3}}{\alpha^{\prime}}
$$

so that as $\alpha^{\prime} \rightarrow 0, k, \rho_{2}, \rho_{3}$ all go to zero exponentially. Again all $\alpha^{\prime}$ dependence disappears from the measure and after using momentum conservation the resulting amplitude as $\alpha^{\prime} \rightarrow 0$ can be written as

$$
\begin{gathered}
A_{3}^{\mathrm{NP}}\left(p_{1} ; p_{2}, p_{3}\right)=\frac{g_{3}^{3}}{8} \frac{\mathrm{e}^{-\frac{\mathrm{i}}{2} p_{2} \theta p_{3}}}{(4 \pi)^{d / 2}} \int_{0}^{\infty} \frac{d T}{T^{d / 2}} \mathrm{e}^{-m^{2} T} \int_{0}^{T} d t_{2} \int_{0}^{T-t_{2}} d t_{3} \exp \left[p_{1} \cdot p_{2} t_{2}\left(1-\frac{t_{2}}{T}\right)\right. \\
\left.+p_{1} \cdot p_{3}\left(t_{2}+t_{3}\right)\left(1-\frac{t_{2}+t_{3}}{T}\right)+p_{2} \cdot p_{3} t_{3}\left(1-\frac{t_{3}}{T}\right)+\frac{1}{4 T} p_{1} \theta^{2} p_{1}-i p_{1} \theta p_{3} \frac{t_{3}}{T}\right]
\end{gathered}
$$

where we denote $p_{1} \theta p_{3} \equiv p_{1}^{\mu} \theta_{\mu \nu} p_{3}^{\nu}$ etc. This result exactly equals the field theory amplitude for the nonplanar Feynman diagram with $p_{2}$ and $p_{3}$ being non-planar, as shown in the Fig 1 . The corresponding planar amplitude with the same cyclic ordering, is eas-

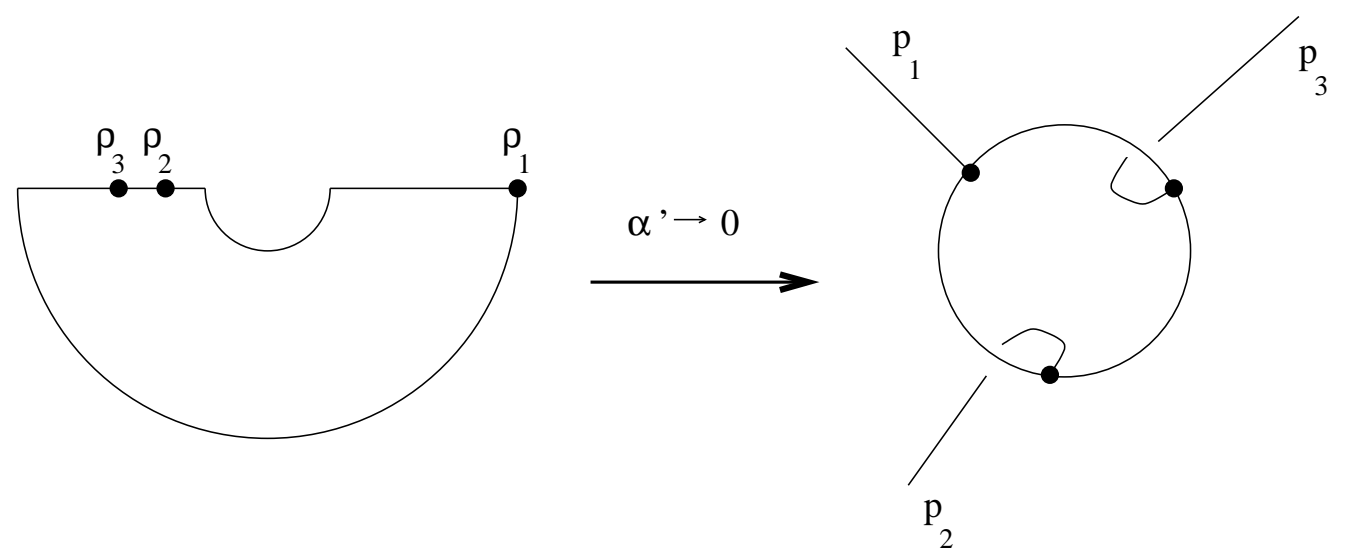

Figure 1: Field theory limit of a nonplanar amplitude.

ily obtained from (3.15) by dropping all $\theta$-dependent terms from the exponent inside the integral, but keeping the factor $e^{-\frac{i}{2} p_{2} \theta p_{3}}$ in front of the integral. All the other six amplitudes are obtained in a completely analogous way and we conclude that the oneloop string amplitudes correctly reproduce all the eight 3-point one-loop diagrams of the noncommutative $\phi^{3}$ field theory.

We could now go on and derive higher-point one-loop amplitudes of the noncommutative $\phi^{3}$ theory from the string amplitudes but it is pretty clear how this will work and that it gives the correct result.

$\phi^{4}$ in 4 dimensions 
It is now interesting to show that the same string master formula (3.2) used for $\phi^{3}$ also correctly reproduces the one-loop amplitudes of noncommutative $\phi^{4}$ theory in $d=4$. We follow [28, 29, 40] where 4-point vertices are obtained by a different scaling of the string moduli. Again we start by fixing the new relation between the string coupling constant and the one appearing in the field theory lagrangian

$$
L_{4}=\frac{1}{2} \partial_{\mu} \phi \partial^{\mu} \phi-\frac{1}{2} m^{2} \phi^{2}+\frac{1}{4 !} g_{4}(\phi * \phi * \phi * \phi) .
$$

Comparing the 4-point vertex a tree level one can read

$$
g_{4}=4 !\left(2 \alpha^{\prime}\right)^{(d-4) / 2} g_{\mathrm{op}}^{2} .
$$

where one can immediately check that the critical dimension of $\phi^{4}$ is correctly reproduced. Since now $g_{\text {op }}$ will bring in (3.2) less powers of $\alpha^{\prime}$, in order to have a finite one-loop amplitude in the field theory limit, one has to consider a limit in which the distance between two cubic vertices is vanishing as $\alpha^{\prime} \rightarrow 0$.

A first simple example is to check how the non-planar $\phi^{4} 2$-point function follows from (3.2). In the field theory diagram we have only one Schwinger parameter which is related in the usual way to the length of the string annulus through (3.7). No more rescaling of string moduli are needed, since the overall factor is now independent of $\alpha^{\prime}$ and thus we shrink to zero the second propagator which would have been present in the $\phi^{3} 2$ point: $-1<<\rho_{2} \rightarrow 0$, but with $\alpha^{\prime} \ln \left|\rho_{2}\right| \rightarrow 0$. As expected, this corner of the region of integration gives the correctly normalized non-planar diagram

$$
A_{2}^{\mathrm{NP}}\left(p_{1},-p_{1}\right)=\frac{g_{4}}{6} \frac{1}{(4 \pi)^{d / 2}} \int_{0}^{\infty} \frac{d T}{T^{d / 2}} \mathrm{e}^{-m^{2} T} \exp \left(p_{1}^{\mu} p_{1}^{\nu} \frac{\theta_{\mu \nu}^{2}}{4 T}\right) .
$$

The planar result is similar to the above equation; a first difference, of course, is the absence of the $\theta$ dependent factor; however in this case also the overall coefficient is different and the factor of $1 / 6$ is replaced by $1 / 3$. As already noticed in the commutative case also, this relative factor of $1 / 2$, between the planar and non-planar contribution, is reproduced by string theory 28, 29]. In fact in the planar amplitude, on can consider the region of $\rho_{2}\left(\rho_{2} \rightarrow 1\right)$ which gives in the $\phi^{3}$ case the tadpole diagram. Again it is possible to shrink the reducible propagator to zero and produce the same $\phi^{4}$ diagram. Notice that this possibility is allowed only for planar diagram and thus in this case the final result is doubled.

In the next examples we obtain the four point one-loop diagrams where $p_{1}$ and $p_{2}$ enter the loop at one vertex and $p_{3}$ and $p_{4}$ at the other, with $p_{1}, p_{2}, p_{3}, p_{4}$ ordered clockwise. In this case one has to scale the moduli $\rho_{2}, \rho_{3}, \rho_{4}$ in such a way that no Schwinger proper time is associated to $\rho_{2} / \rho_{1} \equiv \rho_{2}$ or to $\rho_{3} / \rho_{4}$. Thus, for the planar diagram, we start with the planar open-string four-point one-loop amplitude $A_{4}^{P}$ and do the following scalings

$$
-\alpha^{\prime} \ln \rho_{3}=t_{3}, \quad \frac{\rho_{4}}{\rho_{3}} \text { and } \rho_{2}<<1 \text {, but finite, }
$$


implying also

$$
-\alpha^{\prime} \ln \rho_{4}=t_{3}+\mathcal{O}\left(\alpha^{\prime}\right), \quad-\alpha^{\prime} \ln \rho_{2} \rightarrow 0 .
$$

From (3.2) we then get for the planar 4-point amplitude

$$
\begin{aligned}
A_{4}^{\mathrm{P}}\left(p_{1}, \ldots p_{4}\right) & =\frac{g_{4}^{2}}{36} \frac{1}{(4 \pi)^{d / 2}} \int_{0}^{\infty} \frac{d T}{T^{d / 2}} \mathrm{e}^{-m^{2} T} \int_{\mathrm{e}^{-T / \alpha^{\prime}}}^{1} \frac{d \rho_{2}}{\rho_{2}} \int_{-\alpha^{\prime} \ln \rho_{2}}^{T} d t_{3} \int_{\mathrm{e}^{-T / \alpha^{\prime}}}^{\mathrm{e}^{-t} / \alpha^{\prime}} \frac{d \rho_{4}}{\rho_{4}} \\
& \times \exp \left[\sum_{i<j} 2 \alpha^{\prime} p_{i}^{\mu} p_{j}^{\nu} G_{\mu \nu}^{P}\left(\rho_{i}, \rho_{j}\right)\right] .
\end{aligned}
$$

Note again that all factors of $\alpha^{\prime}$ have canceled from the measure. Since $\widetilde{\rho}_{4} \equiv \rho_{4} / \rho_{3}$ is finite it is more convenient to replace

$$
\int_{\mathrm{e}^{-T / \alpha^{\prime}}}^{\mathrm{e}^{-t_{3} / \alpha^{\prime}}} \frac{d \rho_{4}}{\rho_{4}} \ldots \rightarrow \int_{\mathrm{e}^{-\left(T-t_{3}\right) / \alpha^{\prime}}}^{1} \frac{d \widetilde{\rho}_{4}}{\widetilde{\rho}_{4}} \ldots
$$

so that as $\alpha^{\prime} \rightarrow 0$ we get

$$
\begin{gathered}
A_{4}^{\mathrm{P}}\left(p_{1}, \ldots p_{4}\right)=\frac{g_{4}^{2}}{36} \frac{1}{(4 \pi)^{d / 2}} \int_{0}^{\infty} \frac{d T}{T^{d / 2}} \mathrm{e}^{-m^{2} T} \int_{0}^{1} \frac{d \rho_{2}}{\rho_{2}} \int_{0}^{T} d t_{3} \int_{0}^{1} \frac{d \widetilde{\rho}_{4}}{\widetilde{\rho}_{4}} \\
\quad \exp \left[-\left(p_{1}+p_{2}\right)^{2} t_{3}\left(1-\frac{t_{3}}{T}\right)-\frac{\mathrm{i}}{2}\left(p_{1} \theta p_{2}+p_{3} \theta p_{4}\right)\right] .
\end{gathered}
$$

The integrals on $\rho_{2}$ and $\widetilde{\rho}_{4}$ are divergent in the $\alpha^{\prime} \rightarrow 0$ limit and are handled much as we did with divergence in $k$ [29], that is we write $1 / \rho_{2}=\mathrm{e}^{-\frac{1}{\alpha^{\prime}} \alpha^{\prime} \ln \rho_{2}} \rightarrow \mathrm{e}^{m^{2} \alpha^{\prime} \ln \rho_{2}} \rightarrow 0$ as $\alpha^{\prime} \rightarrow 0$ and similarly for $1 / \tilde{\rho}_{2}$. Hence the integrals simply give 1 and we finally get

$$
A_{4}^{\mathrm{P}}\left(p_{1}, \ldots p_{4}\right)=\frac{g_{4}^{2}}{36} \frac{1}{(4 \pi)^{d / 2}} \mathrm{e}^{-\frac{i}{2}\left(p_{1} \theta p_{2}+p_{3} \theta p_{4}\right)} \int_{0}^{\infty} \frac{d T}{T^{d / 2}} \mathrm{e}^{-m^{2} T} \int_{0}^{T} d t_{3} \mathrm{e}^{\left[-\left(p_{1}+p_{2}\right)^{2} t_{3}\left(1-\frac{t_{3}}{T}\right)\right]} .
$$

Just like the two-point amplitude, one can also get contributions from other regions of the string moduli in the present case. It is easy to see that the reducible $\phi^{3}$ diagrams may degenerate giving the desired $\phi^{4}$ diagram: this can be done in three different ways according to where are the reducible propagators in the original cubic diagram. One can also reproduce the integrand of (3.23) from regions different from the one considered in (3.19). For instance, it is possible to exchange the relative order of the punctures $\rho_{3}$ and $\rho_{4}$ and obtain the same result written above with $p_{3}$ and $p_{4}$ exchanged. Notice however that the two contributions come from the common boundary of two contiguous regions $\left(\rho_{3}<\rho_{4}\right.$ and $\left.\rho_{4}<\rho_{3}\right)$, so that it is natural to weight each of them with a factor of one half [29]. A similar observation holds also for the punctures $\rho_{1}$ and $\rho_{2}$ once one remembers that the points 1 and $k$ of the Schottky parameterization are identified and mapped to a single point on the annulus. Since we have pinched together two pairs of 
external legs, this brings a factor of $1 / 4$ which exactly cancels the "degeneracy" coming from the contributions related to reducible $\phi^{3}$ diagrams and (3.23) really represents the final result for the diagram under study.

Next we look at the same 4 -point function but with " $p_{3}$ and $p_{4}$ nonplanar", i.e. putting the vertex on the inner border of the loop rather than on the outer one. Then one has to start with the non-planar string amplitude with $\rho_{1}=1$ and $\rho_{2}$ on one boundary and $\rho_{3}, \rho_{4}$ on the other, i.e. $\rho_{3}, \rho_{4} \in[-1,-k]$. Inserting absolute values and signs at the appropriate places, the scalings (3.19), (3.20) remain the same. The final result equals (3.23) with the phase factor $e^{-\frac{i}{2}\left(p_{1} \theta p_{2}+p_{3} \theta p_{4}\right)}$ replaced by $e^{-\frac{i}{2}\left(p_{1} \theta p_{2}-p_{3} \theta p_{4}\right)}$ and with an additional factor $\mathrm{e}^{\frac{1}{4 T}\left(p_{3}+p_{4}\right) \theta^{2}\left(p_{3}+p_{4}\right)}$ inserted in the integrand, again in perfect agreement with the field theory result. Following the above arguments, one can also easily work out the global coefficient by adding again the three contribution coming from the regions related to the reducible $\phi^{3}$ diagrams.

Our last scalar example is this same 4-point diagram but now with only " $p_{3}$ being nonplanar". Going through the same steps as before, one is led to

$$
\begin{gathered}
A_{4}^{\mathrm{NP}}\left(p_{1}, p_{2} ; p_{3} ; p_{4}\right)=\frac{g_{4}^{2}}{36} \frac{1}{(4 \pi)^{d / 2}} \mathrm{e}^{-\frac{i}{2}\left(p_{1} \theta p_{2}-p_{3} \theta p_{4}\right)} \int_{0}^{\infty} \frac{d T}{T^{d / 2}} \mathrm{e}^{-m^{2} T} \int_{0}^{T} d t_{3} \\
\exp \left[-\left(p_{1}+p_{2}\right)^{2} t_{3}\left(1-\frac{t_{3}}{T}\right)+\frac{1}{4 T} p_{3} \theta^{2} p_{3}-i p_{3} \theta p_{4} \frac{t_{3}}{T}\right] .
\end{gathered}
$$

From the string point of view, it is also quite easy to fix the global normalization by counting how many contributions one can get from the reducible $\phi^{3}$ diagrams. As is clear in 't Hooft's double line notation, two legs can be pulled away and form a reducible propagator only if they are on the same border. This is also accounted for in string theory where reducible diagrams are related to the singularity $\ln \left|z_{i}-z_{j}\right|$ [23] present only in the planar case. Thus for this diagram one can get only an additional contribution beside the one already computed, so that the final result is twice the one reported in (3.24).

A small remark is due at this point. Contrary to what one expects, the results obtained above for scalar interaction do not seem to be real because of the presence of phase factors. However one has to remember that the physically meaningful quantity is the whole amplitude which contains also the contribution coming from the non-cyclical permutation of the diagrams calculated here. It is easy to see that in the sum the linear terms in $\theta$ disappear and the final result is real. However, this observation does not apply to the last diagram we computed; in fact, in this case, the punctures $\rho_{3}$ and $\rho_{4}$ stay on different boundaries and thus can not be exchanged without changing the topology of the diagram. This means that the final result has to be real by itself, except for the factor $e^{-\frac{i}{2} p_{1} \theta p_{2}}$. One can check this by doing the change of variables $t_{3} \rightarrow T-t_{3}$ in (3.24) whose effect is exactly to switch the signs of the $p_{3} \theta p_{4}$ terms. Of course one or the other form is obtained through a direct field theory computation. 


\section{$3.2 \quad$ Noncommutative photon}

Next we study the noncommutative gauge theory with the action

$$
S=-\frac{1}{4} \int F_{\mu \nu} * F^{\mu \nu}
$$

where $F_{\mu \nu}=\partial_{\mu} A_{\nu}-\partial_{\nu} A_{\mu}+i g\left[A_{\mu}, A_{\nu}\right]_{*}$. The master formula for reproducing the "gluon" amplitude can be easily derived from (2.1) by the usual trick of shifting the external momenta by $p_{i}^{\mu} \rightarrow p_{i}^{\mu}+V_{i}^{\prime}(0) \epsilon_{i}^{\mu} \partial_{\rho_{i}}$ and then isolating from the result the part linear in all $\epsilon_{i}$. We obtain

$$
\begin{aligned}
A_{M}^{(1)}\left(p_{1}, \ldots, p_{M}\right) & =\frac{\left(\sqrt{2} g_{\mathrm{op}}\right)^{M}}{(4 \pi)^{d / 2}}\left(2 \alpha^{\prime}\right)^{(M d-2 M-2 d) / 4} \int_{0}^{1} \frac{d k}{k^{2}}\left(-\frac{\ln k}{2}\right)^{-d / 2} \prod_{n=1}^{\infty}\left(1-k^{n}\right)^{2-d} \\
& \times \int d \rho_{2} \cdots \int d \rho_{M} \exp \left[\sum_{i<j} 2 \alpha^{\prime} p_{i}^{\mu} p_{j}^{\nu} G_{\mu \nu}\left(\rho_{i}, \rho_{j}\right)\right] \\
& \times\left[\exp \sum_{i \neq j}\left(\sqrt{2 \alpha^{\prime}} \epsilon_{i}^{\mu} \partial_{\rho_{i}} G_{\mu \nu}\left(\rho_{i}, \rho_{j}\right) p_{j}^{\nu}+\frac{1}{2} \epsilon_{i}^{\mu} \partial_{\rho_{i}} \partial_{\rho_{j}} G_{\mu \nu}\left(\rho_{i}, \rho_{j}\right) \epsilon_{j}^{\nu}\right)\right]_{\mathrm{m} . \mathrm{l} .},
\end{aligned}
$$

where the subscript "m.l." stands for multilinear, meaning that only terms linear in each polarization should be kept. As usual, we have used the worldsheet projective invariance to fix $\delta=0, \xi \rightarrow \infty$ and $\rho_{1}=1$. Note that the measure for the punctures differs from the one of scalar amplitude in the expected way.

The identification of the string coupling with the gluon coupling can be determined by comparing the tree level field theory 3-point function with the tree level string 3-point amplitude and the result is

$$
g=\sqrt{2} g_{o p}\left(2 \alpha^{\prime}\right)^{(d-4) / 4} .
$$

Before we start doing computations using this master formula, we would like to comment on the $U(1)$ (non)decoupling in a (non)commutative $U(N)$ Yang-Mills theory from the point of view of string theory. In the commutative case, the $U(1)$ factor is free and decouples. As it should be, this can be accounted for in string theory. For example, there is no 3-point tree level amplitude in field theory. The way string theory produces this fact is quite simple, the disc diagram with 3 vertex operators inserted in one order cancels the diagram with the vertex operators inserted in the reversed order. This is a consequence of the fact that vector states of the open bosonic theory carry a quantum number -1 under the world-sheet parity operator $\Omega$. Thus a 3 -point interaction among "photons" is forbidden by the conservation of this quantum number. Note that this symmetry argument not only implies that the 3-point tree level amplitude is zero, but also forbids the presence of internal 3-"photon" vertices in more complicated diagrams. At the loop level, it has been shown in detail [23] that string theory again reproduces 
the one-loop correction to the 3 and 4-point vertex of Yang-Mills. It is instructive to briefly review what is the mechanism that implements the $U(1)$ decoupling at the one loop. To understand this, we consider the simple case of a 2-point amplitude where only one cyclical order of the external legs is possible. A vanishing result is now assured by the cancellation among diagrams with a different number of legs on the inner and outer border of the annulus. The reason is quite clear: due to the form of the vector string state, each vertex brings in the amplitude a derivative of the Green function with respect of the insertion point of the interaction. Now since the field theory limit of the Green function is an even function $\left(G_{\mu \nu}(\rho)=G_{\mu \nu}(-\rho)\right)$, diagrams with legs on different borders will differ simply by a change of sign. In the 2-point case this implies that the planar and the non-planar cancel each other to ensure the $U(1)$ decoupling, even if they are not separately zero. In the noncommutative case, the $U(1)$ factor is not free any more and does not decouple. This non-decoupling can again be accounted for in string theory: the various diagrams, which used to cancel each other in the $\theta=0$ case, are now dressed up with different $\theta$ dependent phases and do not cancel anymore. Thus it is clear that the presence of a noncommutative parameter $\theta$ has the same effect as the Chan-Paton trace in the $S U(N)$ case, and it is no surprise to see that a $\theta$ dependent factor here plays the same role as the $S U(N)$ structure constant.

After this comment on the interaction of $U(1)$, we are ready to do the computation. We will analyze the gluon 2-point amplitude as an example. Restricting (3.26) to the case $M=2$, the gluon 2-point amplitude is given by

$$
\begin{aligned}
& A_{2}\left(p_{1}, p_{2}\right)=\frac{\left(\sqrt{2} g_{o p}\right)^{2}}{(4 \pi)^{d / 2}}\left(2 \alpha^{\prime}\right)^{-1} \int_{0}^{1} \frac{d k}{k^{2}}\left(-\frac{\ln k}{2}\right)^{-d / 2} \prod_{n=1}^{\infty}\left(1-k^{n}\right)^{2-d} \int d \rho^{\prime} \times \\
& \left.\mathrm{e}^{-2 \alpha^{\prime} p_{i} G^{i j}\left(\rho, \rho^{\prime}\right) p_{j}}\left[\epsilon_{1 i} \frac{\partial}{\partial \rho} \frac{\partial}{\partial \rho^{\prime}} G^{i j} \epsilon_{2 j}-2 \alpha^{\prime}\left(\epsilon_{1 i} \frac{\partial}{\partial \rho} G^{i j} p^{j}\right)\left(p^{i} \frac{\partial}{\partial \rho^{\prime}} G^{i j} \epsilon_{2 j}\right)\right]\right|_{\rho=1},
\end{aligned}
$$

where $G^{i j}$ is the Green function (2.41) or (2.43) depending on whether we are considering the planar diagram or the nonplanar diagram; the integration region for $\rho^{\prime}$ is $(k, 1)$ in the first case, while in the latter $\rho^{\prime} \in(-1,-k)$. In order to perform the field theory limit, as before, one has to introduce the Schwinger parameters

$$
k=\mathrm{e}^{-T / \alpha^{\prime}}, \quad \rho^{\prime}=-\mathrm{e}^{-t / \alpha^{\prime}}
$$

with $t, T$ fixed. The modification in the planar case is simple, the additional term in the Green function is a step function (2.41). In general one should drop the contribution coming from the derivatives of the step function, as it corresponds to put two vertex operators at the same point, which is not allowed in string interaction. Therefore the only modification to the planar $M$ gluon amplitude is the usual phase factor.

The nonplanar case is more interesting. Using (2.43) and (2.27), we arrive at

$$
A_{2}(p,-p)=\frac{\left(\sqrt{2} g_{o p}\right)^{2}}{(4 \pi)^{d / 2}}\left(2 \alpha^{\prime}\right)^{-1} \int_{0}^{1}[d k] \int_{-1}^{-k} d \rho^{\prime} \mathrm{e}^{-2 \alpha^{\prime} p^{2} I_{0}-\frac{(\theta p)^{2}}{4 T}} J,
$$


where $I_{0}$ is given by (2.27), $[d k]$ is the measure

$$
[d k]=\frac{d k}{k^{2}}\left(-\frac{\ln k}{2}\right)^{-d / 2} \prod_{n=1}^{\infty}\left(1-k^{n}\right)^{2-d},
$$

and $J=J_{0}+J_{1}+J_{2}$ are the polarization dependent pieces in (3.28)

$$
\begin{gathered}
J_{0}=\epsilon_{1} \cdot \epsilon_{2} \frac{\partial^{2} I_{0}}{\partial \rho \partial \rho^{\prime}}-2 \alpha^{\prime}\left(\epsilon_{1} \cdot p\right)\left(\epsilon_{2} \cdot p\right) \frac{\partial I_{0}}{\partial \rho} \frac{\partial I_{0}}{\partial \rho^{\prime}}, \\
J_{1}=\frac{i}{\ln k}\left[\frac{\partial I_{0}}{\partial \rho^{\prime}}\left(\epsilon_{1} \theta p\right)\left(\epsilon_{2} \cdot p\right)+\frac{1}{\rho^{\prime}} \frac{\partial I_{0}}{\partial \rho}\left(\epsilon_{2} \theta p\right)\left(\epsilon_{1} \cdot p\right)\right], \\
J_{2}=\frac{-1}{2 \alpha^{\prime}(\ln k)^{2}} \frac{1}{\rho^{\prime}}\left(\epsilon_{1} \theta p\right)\left(\epsilon_{2} \theta p\right) .
\end{gathered}
$$

where the subscript of $J$ denotes the powers of $\theta$.

The 1-loop amplitude, as computed in (3.28), contains the propagation of all the string states within the loop. This is partially reflected in the fact that the measure contains all powers of $k$. As explained in [23], the contribution from the gluon (which is at level 1 of the open string spectrum) can be identified with the contribution coming from the pieces linear in $k$ in the integrand (3.28). It is straightforward to do the expansion in $k$ in order to isolate the relevant factors

$$
\begin{gathered}
\int_{0}^{1}[d k]=2\left(2 \alpha^{\prime}\right)^{d / 2-1} \int_{0}^{\infty} \frac{d T}{T^{d / 2}}\left[\frac{1}{k}+(d-2)+\cdots\right], \\
\frac{\partial I_{0}}{\partial \rho^{\prime}}=\frac{1}{\rho^{\prime}}\left(\frac{t}{T}-\frac{1}{2}-\rho^{\prime}+\frac{k}{\rho^{\prime}}-k \rho^{\prime}+O\left(k^{2}\right)\right)=-\frac{1}{\rho^{\prime}} \frac{\partial I_{0}}{\partial \rho} .
\end{gathered}
$$

If we now pass from the string moduli to the Schwinger parameters by means of (3.29), we get

$$
A_{2}(p,-p)=\frac{2}{\alpha^{\prime}} \frac{g^{2}}{(4 \pi)^{d / 2}} \int_{0}^{\infty} \frac{d T}{T^{d / 2}}\left[\frac{1}{k}+(d-2)+\cdots\right] \int_{0}^{T} d t \mathrm{e}^{-\left(2 \alpha^{\prime}\right) p^{2} I_{0}-\frac{(\theta p)^{2}}{4 T}}\left(\rho^{\prime} J\right) .
$$

Let us focus now on the contribution which is linear in $\theta$

$$
J_{1} \sim \frac{-\mathrm{i}}{\ln k} \frac{1}{\rho^{\prime}}\left(\frac{t}{T}-\frac{1}{2}-\rho^{\prime}+\frac{k}{\rho^{\prime}}-k \rho^{\prime}+\cdots\right) .
$$

and since $-\rho^{\prime}+\frac{k}{\rho^{\prime}}-k \rho^{\prime} \rightarrow 0$, so the contribution of $J_{1}$ in the two point function is

$$
\begin{aligned}
A_{2}\left(J_{1}\right) & =-2 \mathrm{i}(d-2) \frac{g^{2}}{(4 \pi)^{d / 2}}\left[\left(\epsilon_{1} \theta p\right)\left(\epsilon_{2} \cdot p\right)-\left(\epsilon_{2} \theta p\right)\left(\epsilon_{1} \cdot p\right)\right] \\
& \times \int_{0}^{\infty} \frac{d T}{T^{d / 2+1}} \int_{0}^{T} d t\left(\frac{t}{T}-\frac{1}{2}\right) \mathrm{e}^{-p^{2}\left(t-\frac{t^{2}}{T}\right)-\frac{(\theta p)^{2}}{4 T}} .
\end{aligned}
$$


It is easy to see that the integral of $t$ is is a total derivative and integrates to zero. Therefore the linear term $J_{1}$ is vanishing.

$$
A_{2}\left(J_{1}\right)=0 .
$$

As for $J_{0}$, one can do an integration by part on $\rho^{\prime}$ and it is easy to see that one reproduces the usual tensor structure for the 2-point amplitude. Thus the explicit form of the $J_{0}$ contibution is

$$
\begin{aligned}
A_{2}\left(J_{0}\right) & =-4 \frac{g^{2}}{(4 \pi)^{d / 2}}\left[\left(\epsilon_{1} \epsilon_{2}\right) p^{2}-\left(\epsilon_{1} \cdot p\right)\left(\epsilon_{2} \cdot p\right)\right] \\
& \times \int_{0}^{\infty} \frac{d T}{T^{d / 2}} \int_{0}^{T} d t\left[\left(\frac{t}{T}-\frac{1}{2}\right)^{2}(d-2)-2\right] \mathrm{e}^{-p^{2}\left(t-\frac{t^{2}}{T}\right)-\frac{(\theta p)^{2}}{4 T}} .
\end{aligned}
$$

For $d=4$, the integral is

$$
\frac{1}{2} \int_{0}^{\infty} \frac{d T}{T} \mathrm{e}^{-\frac{\tilde{p}^{2}}{4 T}} \int_{0}^{1} d x \mathrm{e}^{-T p^{2} x(1-x)}\left[(1-2 x)^{2}-4\right]=\int_{0}^{1} d x\left[(1-2 x)^{2}-4\right] K_{0}(\sqrt{x(1-x)}|p||\tilde{p}|) .
$$

Now the leading divergence for the Bessel function is

$$
K_{0}(x) \sim \ln \frac{2}{x}, \quad x \rightarrow 0 .
$$

So we obtain

$$
A_{2}\left(J_{0}\right)=\frac{g^{2}}{(4 \pi)^{d / 2}}\left(\frac{22}{3} \ln \left(|p|^{2}|\tilde{p}|^{2}\right)+\cdots\right)\left[\left(\epsilon_{1} \epsilon_{2}\right) p^{2}-\left(\epsilon_{1} \cdot p\right)\left(\epsilon_{2} \cdot p\right)\right] .
$$

Notice that there is a $\ln \theta$-type singularity.

Finally we look at $J_{2}$, which is more interesting. It is

$$
J_{2}=-\frac{\alpha^{\prime}}{2 T^{2}} \frac{1}{\rho^{\prime}}\left(\epsilon_{1} \theta p\right)\left(\epsilon_{2} \theta p\right),
$$

and hence it is contribution in $A_{2}$ is

$$
A_{2}\left(J_{2}\right)=-(d-2) \frac{g^{2}}{(4 \pi)^{d / 2}} \int_{0}^{\infty} \frac{d T}{T^{d / 2+2}} \int_{0}^{T} d t \mathrm{e}^{-p^{2}\left(t-\frac{t^{2}}{T}\right)-\frac{(\theta p)^{2}}{4 T}}\left(\epsilon_{1} \theta p\right)\left(\epsilon_{2} \theta p\right) .
$$

For $d=4$, the integral is equal to

$$
\begin{aligned}
\int_{0}^{\infty} \frac{d T}{T^{3}} \mathrm{e}^{-\frac{(\theta p)^{2}}{4 T}} \int_{0}^{1} d x \mathrm{e}^{-T p^{2} x(1-x)} & =\left.\frac{16}{\tilde{p}^{4}} \cdot \frac{\partial^{2}}{\partial a^{2}}\left(\int_{0}^{\infty} \frac{d T}{T} \mathrm{e}^{-\frac{a(\tilde{p})^{2}}{4 T}} \int_{0}^{1} d x \mathrm{e}^{-T p^{2} x(1-x)}\right)\right|_{a=1} \\
& =\left.\frac{32}{\tilde{p}^{4}} \cdot \frac{\partial^{2}}{\partial a^{2}} \int_{0}^{1} d x K_{0}(\sqrt{x(1-x)}|p||\tilde{p}| \sqrt{a})\right|_{a=1}
\end{aligned}
$$


By expanding the above formula for small momenta, we can extract the leading IR behavior of $J_{2}$

$$
A_{2}\left(J_{2}\right)=\frac{2 g^{2}}{\pi^{2}} \frac{\left(\epsilon_{1} \tilde{p}\right)\left(\epsilon_{2} \tilde{p}\right)}{\tilde{p}^{4}}+\cdots
$$

This form of $1 / \theta^{2}$ singularity in the two gluon amplitude was first obtained in [14 and is another example of $\mathrm{UV} / \mathrm{IR}$ mixing in noncommutative field theory which was stressed in [10]. Also in the 4 dimensional scalar $\phi^{4}$ theory the leading singularity is of the form $1 /(\theta p)^{2}$ as one can easily see from the result of the nonplanar 2-point function (3.18). Since the commutative gauge theory is less divergent in the UV than the commutative scalar theory, one could have expected that the leading singularity in the noncommutative gauge theory is of the form $\ln (\theta p)^{2}$. However, as was shown in [14, 15], this expectation is not correct and one still gets the $1 /(\theta p)^{2}$ behavior. As pointed out in [15], the appearance of the $1 /(\theta p)^{2}$ piece does not spoil gauge invariance. In fact it does not correspond to a mass shift because of its unusual tensor structure. Here we have re-derived this result using string theory. In addition, we have been able to determine also the subleading singularity from string theory which is of the form $\ln (\theta p)^{2}$ from string theory.

Summarizing, we have

$$
A_{\mathrm{NP}}=\frac{g^{2}}{16 \pi^{2}}\left\{32 \frac{\left(\epsilon_{1} \tilde{p}\right)\left(\epsilon_{2} \tilde{p}\right)}{\tilde{p}^{4}}+\frac{22}{3} \ln \left(|p|^{2}|\tilde{p}|^{2}\right)\left[\left(\epsilon_{1} \epsilon_{2}\right) p^{2}-\left(\epsilon_{1} \cdot p\right)\left(\epsilon_{2} \cdot p\right)\right]\right\}+\ldots
$$

where dots stay for terms which are finite when $\theta \rightarrow 0$. It is interesting to note that the whole result comes from a single corner of the moduli space. This is because we performed an integration by parts which has the effect of including the tadpole contribution in the region here considered. We note that the vacuum polarization of the photon was also obtained by Hayakawa [14] in the Feynman gauge. The coefficient of the $\ln \theta$ term is clearly related to the wave-function renormalization constant of the commutative case and thus is gauge dependent. The result found in [14] is thus different from ours, because his calculation was performed in the Feynman gauge. On the contrary, as explained in [23] for the commutative case, string theory amplitudes naturally give rise to field theory results in the background field method [41. Recall that a distinct property of Feynman diagrams in the background field gauge is to leave the gauge invariance of the external particles unbroken. For the noncommutative case, the situation is the same and we conclude that string theory again gives field theory results computed in the background field method. One of the advantages of our result is that the $\beta$ function can be determined directly from the two point function without the need of computing the vertex corrections. By exploiting this and the fact that the planar diagram contribution is exactly the same (apart from a trivial overall group theory factor of $N$ ) as those in a usual commutative $S U(N)$ gauge theory, we easily obtain the 1-loop $\beta$ function

$$
\beta=-\frac{g^{2}}{16 \pi^{2}} \frac{22}{3}
$$


The $\beta$-function for the noncommutative QED has also been computed in [13] [14] in the Feynman gauge, by combining vertex corrections and wave function renormalization. The Yang-Mills $\beta$-function can be elegantly derived also from the string generating functional by following the approach of Mastaev and Tseytlin [42]. Again, the noncommutative paramenter $\theta$ can be easily implemented in the string calculation by switching on a constant magnetic field. It is easy to see that the derivation of 42 is not substantially modified and one finds the distinctive factor $26-D$ : this is, from this point of view, the source of the Yang-Mills $\beta$-function $\sim 22 / 3$.

\section{Discussion}

In this paper, we used the boundary state approach to compute the one-loop open string Green function by calculating the string amplitude with two boundaries inserted. We note that generally there is an ambiguity in the resulting Green function that cannot be fixed from the boundary state approach. We pointed out that the scalar amplitudes do not care about this ambiguity. However, this ambiguity can be fixed by simply comparing with the planar gluon 2-point function and one obtains the correct open string Green function which can then be used in the string master formula in a uniform manner for all mass levels. A more satisfactory way 43] to obtain the desired Green function would be to calculate directly the open string scattering amplitudes using the vertex operators constructed from the open string modes (2.35)-(2.37) and extract from there the Green function.

An interesting behavior of noncommutative quantum field theory is that although classically the noncommutative description is a smooth deformation of the commutative description, quantum mechanically they are different and generally one cannot turn off $\theta$ to smoothly reduce back to the commutative description. The UV/IR mixing [10 and the induced Chern-Simons action in odd dimensions [18] are examples. The fact that a commutative field theory differ intrinsically from a noncommutative field theory is clear from the present approach. It is important to recall that in deriving the noncommutative field theory limit from string theory, one has to take the double scaling limit (1.4) with $\alpha^{\prime} \rightarrow 0, F \rightarrow \infty$ and $\alpha^{\prime} F$ fixed in order to have a nonzero $\theta$. The commutative case $\theta=0$, however, is obtained from a different limit with $F$ fixed instead and it is thus clear that the commutative and the noncommutative field theories thus obtained differ in character.

We now discuss some possible further directions of work. In this paper, we have shown that by taking the limit (1.4), (1.5) appropriately, one can obtain field theory results from a single string master formula in a uniform manner. We presented explicit calculations for both the case of noncommutative scalar theories and noncommutative gauge theory

\footnotetext{
${ }^{5}$ We would like to thank A. Tseytlin for pointing out this possibility to us.
} 
with gauge group $U(1)$. However, it is straightforward to add Chan-Paton factors and obtain results for noncommutative field theory with gauge group $U(N)$. One difference is that in the $U(N)$ case, the action is no longer even in $\theta$ and one will get field theory result with odd power dependence in $\theta$ also. This can definitely be accounted for by the Green function we obtained. Another possible generalization is to consider superstring amplitudes in the presence of $F$-field and to extract the field theory limit. The field theory is of course a noncommutative supersymmetric one. It may be interesting to understand some of the field theory issues (like non-renormalization theorem and duality for noncommutative field theory) from the string point of view. Also, it is possible to generalize all this to higher loops [43] and we hope to report on some of these topics in the future.

\section{Acknowledgments}

R. R. wants to thank P. Di Vecchia, M. Frau, A. Lerda and R. Marotta for useful discussions and A. Frizzo and L. Magnea for e-mail exchange. We are also grateful to H. Dorn for helpful correspondence after the submission of the first version of the present paper. This work was partially supported by the Swiss National Science Foundation, by the European Union under TMR contract ERBFMRX-CT96-0045 and by the Swiss Office for Education and Science.

\section{References}

[1] A. Connes, M.R. Douglas, and A. Schwarz, JHEP 02, 003 (1998), hep-th/9711162.

[2] M.R. Douglas and C. Hull, JHEP 02, 008 (1998), hep-th/9711165.

[3] C.-S. Chu and P.-M. Ho, Nucl. Phys. B550, 151 (1999), hep-th/9812219.

[4] V. Schomerus, JHEP 06, 030 (1999), hep-th/9903205.

[5] C.-S. Chu, P.-M. Ho, and M. Li, hep-th/9911153.

[6] N. Seiberg and E. Witten, JHEP 09, 032 (1999), hep-th/9908142.

[7] C.-S. Chu, hep-th/0001144.

[8] A. Abouelsaood, C. G. Callan, C. R. Nappi, and S. A. Yost, Nucl. Phys. B280, 599 (1987).

[9] I. Chepelev and R. Roiban, hep-th/9911098. 
[10] S. Minwalla, M.V. Raamsdonk, and N. Seiberg, hep-th/9912072.

M.V. Raamsdonk and N. Seiberg, hep-th/0002186.

[11] I. Ya. Aref'eva, D. M. Belov, and A. S. Koshelev, hep-th/9912075.

I. Ya. Aref'eva, D. M. Belov, and A. S. Koshelev, hep-th/0001215.

[12] W. Fischler, E. Gorbatov, S. Paban A. Kashani-Poor, P. Pouliot, and J. Gomis, hep-th/0002067.

[13] C. P. Martin and D. Sanchez-Ruiz, Phys. Rev. Lett. 83, 476-479 (1999), hep-th/9903077.

M. M. Sheikh-Jabbari, JHEP 06, 015 (1999), hep-th/9903107.

T. Krajewski and R. Wulkenhaar, hep-th/9903187.

[14] M. Hayakawa, hep-th/9912094.

M. Hayakawa, hep-th/9912167.

[15] A. Matusis, L. Susskind, and N. Toumbas, hep-th/0002075.

[16] J. Madore, S. Schraml, P. Schupp, and J. Wess, hep-th/0001203.

[17] F. Ardalan and N. Sadooghi, hep-th/0002143.

J. M. Gracia-Bondia and C. P. Martin, hep-th/0002171.

L. Bonora, M. Schnabl, and A. Tomasiello, hep-th/0002210.

[18] C.-S. Chu, hep-th/0003007.

[19] Z. Bern and D.A. Kosower, Phys. Rev. Lett. 66, 1669-1672 (1991).

Z. Bern and D. A. Kosower, Nucl. Phys. B379, 451 (1992).

[20] Z. Bern, L. Dixon, and D.A. Kosower, Ann. Rev. Nucl. Part. Sci. 46, 109 (1996), hep-ph/9602280.

[21] Z. Bern and D. A. Kosower, Phys. Rev. D38, 1888 (1988).

[22] P. Di Vecchia, A. Lerda, L. Magnea, and R. Marotta, Phys. Lett. 351B, 445 (1995), hep-th/9502156.

[23] P. Di Vecchia, A. Lerda, L. Magnea, R. Marotta, and R. Russo, Nucl. Phys. B469, 235 (1996), hep-th/9601143.

[24] Z. Bern, David C. Dunbar, and Tokuzo Shimada, Phys. Lett. B312, 277-284 (1993), hep-th/9307001.

Z. Bern, L. Dixon, D. C. Dunbar, M. Perelstein, and J. S. Rozowsky, Nucl. Phys. B530, 401 (1998), hep-th/9802162. 
[25] K. Roland, Phys. Lett. 289 B, 148 (1992).

[26] P. Di Vecchia, A. Lerda, L. Magnea, R. Marotta, and R. Russo, Phys. Lett. B388, 65 (1996), hep-th/9607141.

[27] K. Roland and H. Sato, Nucl. Phys. B485, 241 (1996), hep-th/9604152.

K. Roland and H. Sato, Nucl. Phys. B515, 488 (1997), hep-th/9709019.

L. Magnea and R. Russo, Two loop gluon diagrams from string theory, In Proceedings of the Workshop "Deep Inelastic Scattering '97, Chicago (1997), 1997.

Z. Bern, J.S. Rozowsky, and B. Yan, Phys. Lett. B401, 273 (1997), hep-ph/9702424.

[28] R. Marotta and F. Pezzella, (1999), hep-th/9912158.

[29] A. Frizzo, L. Magnea, and R. Russo, (1999), hep-th/9912183.

[30] L. Cappiello, R. Marotta, R. Pettorino and F. Pezzella, Mod. Phys. Lett. A13 (1998) p. 2433, hep-th/9804032

L. Cappiello, R. Marotta, R. Pettorino and F. Pezzella, Mod. Phys. Lett. A13 (1998) p. 2845, hep-th/9808164;

A. Liccardo, R. Marotta and F. Pezzella, Mod. Phys. Lett. A14 (1999) p. 799, hep-th/9903027.

[31] M. J. Strassler, Nucl. Phys B 385, 145 (1992), hep-ph/9205205.

M. G. Schmidt and C. Schubert, Phys. Lett. B331, 69-76 (1994), hep-th/9403158.

C. Schubert, Acta Phys. Polon. B27, 3965 (1996), hep-th/9610108.

H.-T. Sato and M. G. Schmidt, Nucl. Phys. B524, 742 (1998), hep-th/9802127.

H.-T. Sato and M. G. Schmidt, Nucl. Phys. B560, 551 (1999), hep-th/9812229.

[32] P. Di Vecchia, F. Pezzella, M. Frau, K. Hornfeck, A. Lerda, and S. Sciuto, Nucl. Phys. B322, 317 (1989).

[33] M. Frau, I. Pesando, S. Sciuto, A. Lerda, and R. Russo, Phys. Lett. 400B, 52 (1997), hep-th/9702037.

[34] P. Di Vecchia, M. Frau, I. Pesando, S. Sciuto, A. Lerda, and R. Russo, Nucl. Phys. B507, 259 (1997), hep-th/9707068.

[35] P. Di Vecchia and A. Liccardo hep-th/9912161.

P. Di Vecchia and A. Liccardo hep-th/9912275.

[36] P. Di Vecchia, M. Frau, A. Lerda, and A. Liccardo, hep-th/9906214.

[37] M. B. Green, J. H. Schwarz, and E. Witten, Superstring Theory, Cambridge University Press, 1987.

[38] T. Filk, Phys. Lett. B376, 53 (1996). 
[39] C.-S. Chu and F. Zamora, JHEP 02, 022 (2000), hep-th/9912153.

[40] R. Marotta and F. Pezzella, (1999), hep-th/0003044.

[41] L. F. Abbott, Nucl. Phys. B185, 189 (1981).

[42] R.R. Metsaev, A.A. Tseytlin, Nucl. Phys. B298, 109 (1988) .

[43] work in progress

[44] O. Andreev and H. Dorn, hep-th/0003113.

[45] Y. Kiem and S. Lee, hep-th/0003145.

[46] C.-S. Chu, R. Russo, S. Sciuto, hep-th/0004188. 